\title{
Polytropic dark matter flows illuminate dark energy and accelerated expansion
}

\author{
K. Kleidis ${ }^{1}$ and N. K. Spyrou ${ }^{2}$ \\ ${ }^{1}$ Department of Mechanical Engineering, Technological Education Institute of Central Macedonia, 62124 Serres, Greece \\ e-mail: kleidis@teiser.gr \\ 2 Department of Astronomy, Aristoteleion University of Thessaloniki, 54124 Thessaloniki, Greece \\ e-mail: spyrou@auth.gr
}

Received 13 June 2014 / Accepted 11 November 2014

\begin{abstract}
Currently, a large amount of data implies that the matter constituents of the cosmological dark sector might be collisional. An attractive feature of such a possibility is that, it can reconcile dark matter (DM) and dark energy (DE) in terms of a single component, accommodated in the context of a polytropic-DM fluid. In fact, polytropic processes in a DM fluid have been most successfully used in modeling dark galactic haloes, thus significantly improving the velocity dispersion profiles of galaxies. Motivated by such results, we explore the time evolution and the dynamical characteristics of a spatially-flat cosmological model, in which, in principle, there is no DE at all. Instead, in this model, the DM itself possesses some sort of fluidlike properties, i.e., the fundamental units of the Universe matter-energy content are the volume elements of a DM fluid, performing polytropic flows. In this case, together with all the other physical characteristics, we also take the energy of this fluid's internal motions into account as a source of the universal gravitational field. This form of energy can compensate for the extra energy, needed to compromise spatial flatness, namely, to justify that, today, the total energy density parameter is exactly unity. The polytropic cosmological model, depends on only one free parameter, the corresponding (polytropic) exponent, $\Gamma$. We find this model particularly interesting, because for $\Gamma \leq 0.541$, without the need for either any exotic DE or the cosmological constant, the conventional pressure becomes negative enough so that the Universe accelerates its expansion at cosmological redshifts below a transition value. In fact, several physical reasons, e.g., the cosmological requirement for cold DM (CDM) and a positive velocity-of-sound square, impose further constraints on the value of $\Gamma$, which is eventually settled down to the range $-0.089<\Gamma \leq 0$. This cosmological model does not suffer either from the age problem or from the coincidence problem. At the same time, this model reproduces to high accuracy the distance measurements performed with the aid of the supernovae ( $\mathrm{SNe}$ ) Type Ia standard candles, and most naturally interprets, not only when, but also why the Universe transits from deceleration to acceleration, thus arising as a mighty contestant for a DE model.
\end{abstract}

Key words. dark matter - dark energy

\section{Introduction}

The study of the cosmic microwave background (CMB) has proven to be a powerful tool in exploring the post-recombination Universe. In particular, observations of the temperature variations in the CMB have provided strong evidence that the Universe emerged out of the radiation epoch as a spatially-flat Robertson-Walker (RW) model (see, e.g., de Bernardis et al. 2000; Jaffe et al. 2001; Padin et al. 2001; Stompor et al. 2001; Netterfield et al. 2002; Spergel et al. 2003, 2007; Komatsu et al. 2009, 2011)

This result, however, implies that the overall energy density, $\varepsilon$, of the Universe matter-energy content in units of the quantity $\varepsilon_{\mathrm{c}}=\rho_{\mathrm{c}} c^{2}$ (equivalent to the critical rest-mass density, $\rho_{\mathrm{c}}=\frac{3 H_{0}^{2}}{8 \pi G}$, where $H_{0}$ is the Hubble parameter at the present epoch, $c$ is the velocity of light, and $G$ is Newton's universal constant of gravitation), should be very close to unity, $\Omega=\frac{\varepsilon}{\varepsilon_{\mathrm{c}}} \simeq 1$, i.e., much larger than the measured value of the density parameter, $\Omega_{\mathrm{M}}=\frac{\rho}{\rho_{c}}=0.279$ (Hinshaw et al. 2013). In other words, either an extra amount of energy or a component that has not, yet, been taken into account is needed to compromise spatial flatness.

The need for a distributed extra-energy component, i.e., one that is not associated with concentrations of mass, is also suggested by high-precision distance measurements, performed with the aid of the SNe Ia standard candles (Hamuy et al. 1996; Garnavich et al. 1998; Perlmutter et al. 1998, 1999a; Schmidt et al. 1998; Riess et al. 1998, 2001, 2004, 2007; Knop et al. 2003; Tonry et al. 2003; Barris et al. 2004; Krisciunas et al. 2005; Astier et al. 2006; Jha et al. 2006; Miknaitis et al. 2007; Wood-Vasey et al. 2007; Amanullah et al. 2008, 2010; Holtzman et al. 2008; Kowalski et al. 2008; Hicken et al. 2009a,b; Kessler et al. 2009; Contreras et al. 2010; Guy et al. 2007; Suzuki et al. 2012). In view of these measurements, in a cosmological model with matter content in the form of dust, i.e., of vanishing pressure, the far-off, light-emitting sources appear to be dimmer (i.e., they seem to lie farther away) than what is theoretically expected. In confronting with this result, Riess et al. (1998) and Perlmutter et al. (1999a) admitted that theory (e.g., Carroll et al. 1992) meets observation only in the case of a non zero cosmological constant, $\Lambda$, so that $\Omega_{\mathrm{M}} \simeq 0.3$ and $\Omega_{\Lambda}=\frac{\Lambda c^{2}}{3 H_{0}^{2}} \simeq 0.7$ (see also Riess et al. 2004). A non-vanishing cosmological constant, however, is related to a repulsive component of the gravitational force (see, e.g., Sahni 2004) and, therefore, the apparent dimming of the distant light-emitting sources was subsequently attributed to a (relatively recent) phase of accelerated expansion (see, e.g., Frieman et al. 2008; Linder 2008).

Apart from Enstein's "bigest blunder", the particle-physics vacuum does contribute an effective cosmological constant, 
which could also serve as compensation for the extra energy needed to flatten the Universe (Sahni \& Starobinsky 2000). Unfortunately, the energy density attributed to this source is $10^{123}$ times larger than what is observed (see, e.g., Padmanabhan 2003), hence, an approach other than the cosmological constant could also be useful.

In this context, many physically-motivated models have appeared in the literature, including quintessence (Caldwell et al. 1998), K-essence (Armendariz-Picon et al. 2001), phantom cosmology (Caldwell 2002) and tachyonic matter (Padmanabhan 2002), involving (also) several braneworld scenarios, such as DGP-gravity (Dvali et al. 2000) and the landscape scenario (Bousso \& Polchinski 2000), as well as alternative-gravity theories, such as the scalar-tensor theories (Esposito-Farese \& Polarski 2001) and $f(R)$-gravity (Capozziello et al. 2003), holographic gravity (Cohen et al. 1999; Li 2004; Pavón \& Zimdahl 2005), Chaplygin gas (Kamenshchik et al. 2001; Bento et al. 2002; Bean \& Doré 2003; Sen \& Scherrer 2005), Cardassian cosmology (Freese \& Lewis 2002; Gondolo \& Freese 2003; Wang et al. 2003), theories of compactified internal dimensions (Mongan 2001; Defayet et al. 2002; Perivolaropoulos 2003; Sami et al. 2004), mass-varying neutrinos (Fardon et al. 2004; Peccei 2005), and so on (for a detailed review see, e.g., Caldwell \& Kamionkowski 2009).

In the meantime, the list of observational data in favor of a distributed extra-energy component continued to grow, including evidence from galaxy clusters (Allen et al. 2004), the integrated Sachs-Wolfe (ISW) effect (Boughn \& Crittenden 2004), baryon acoustic oscillations (BAOs, Eisenstein et al. 2005; Percival et al. 2010), weak gravitational lensing (WGL, Huterer 2002; Copeland et al. 2006), and the Lyman- $\alpha$ (LYA) forest (Seljak et al. 2006). A combination of these data with those from the Wilkinson Microwave Anisotropy Probe (WMAP) survey (see, e.g., Dunkley et al. 2009) has provided evidence for cosmic acceleration at the $5 \sigma$ level. This could no longer be ignored, and the 2011 Nobel Prize in Physics officially validated the accelerated expansion of the Universe (in connection, see Riess 2012)

Eventually, both the extra energy needed to compromise spatial flatness and the accelerated expansion of the Universe, which is implemented to justify the unexpected dimming of the SNe Ia standard candles, were reconciled by another assumption: that there is an exotic fluid endowed with negative pressure, which has been termed dark energy (Turner \& White 1997; Perlmutter et al. 1999b), reflecting our ignorance on its exact nature (for a review of the various dark energy (DE) models see, e.g., Miao et al. 2011). However, a dark component (namely, the dark matter, DM, itself) was already present in the energy density of the Universe matter content.

Indeed, it is rather well established that almost $84 \%$ (by mass) of the matter in the Universe consists of nonluminous and nonbaryonic material (see, e.g., Tegmark et al. 2006; Hinshaw et al. 2013). In fact, recent observations based on WGL suggest that the matter distribution of galaxies extends beyond the virial radius, roughly to the middle of the neighboring galaxies (Masaki et al. 2012; in connection; see also Spyrou 2011). This mass distribution could explain the gap, observed in the massdensity profiles, between the global value $\Omega_{\mathrm{M}}=0.279$ and the typical value $\Omega_{\mathrm{gal}}=0.150$, arising from the luminosity density of the galaxies multiplied by the corresponding mass-to-light ratio.

Although we do not know for certain how the DM came to be formed, a sizeable relic abundance of weakly-interacting massive particles (WIMPs) is generally expected to have been produced as a by-product of the Universe's hot youth (see, e.g., Kolb \& Turner 1990, p. 369). These particles decouple from radiation much earlier than pure baryonic matter does. Hence, very soon after recombination $\left(t_{R}\right)$, the baryons fall into deep potential wells of the already evolved DM perturbations and become bounded to them, i.e., for $t>t_{R}$, there are no freely-floating baryons around (see, e.g., Olive 2003; Hooper 2009).

As far as structure formation is concerned, all forms of DM are not equivalent. Particles that are still highly relativistic at $t_{R}$ (such as neutrinos or other particles with masses lower than $100 \mathrm{eV} / c^{2}$ ) have the property that, due to free streaming, erase perturbations out to very large scales (Bond et al. 1980). It is, therefore, expected that very-large-scale structures form first and subsequently fragment to form galaxies later. Particles with this property are termed hot dark matter (HDM). On the other hand, cold dark matter (CDM, i.e., particles with masses larger than $1 \mathrm{MeV} / \mathrm{c}^{2}$ ) has the opposite behavior: small-scale structures form first, aggregating to form larger structures later (Bond \& Szalay 1983). It is now well-known that pure HDM cosmologies can not reproduce the observed large-scale structure of the Universe (see, e.g., Klypin et al. 1993). On the contrary, CDM models are in remarkably good agreement with the observed power spectrum of LYA absorbers (Croft et al. 1999). Apart from debating the precise nature of the DM constituents, the scientific community argues that they should be collisionless.

Many results from the high-energy particle detector PAMELA (Adriani et al. 2009), however, combined with data from the WMAP survey (Hooper et al. 2007), have revealed an unusually high electron - positron production in the Universe, much more than that anticipated by $\mathrm{SNe}$ explosions or cosmicray collisions. These results have led many scientists to argue that among the best candidate sources of these high-energy events are the annihilations of WIMPs (see, e.g., Barger et al. 2008; Bergstrom et al. 2008; Cirelli \& Strumia 2008; Regis \& Ullio 2008; Baushev 2009; Cholis et al. 2009a,b; Fornasa et al. 2009; Fox \& Poppitz 2009; Kane et al. 2009; Zurek 2009), i.e., that the DM constituents can be slightly collisional (Spergel \& Steinhardt 2000; Arkani-Hamed et al. 2009; Cirelli et al. 2009; Cohen \& Zurek 2010; Van den Aarssen et al. 2012), although, there are studies that disagree with this interpretation (see, e.g., Feng et al. 2010). An attractive feature of a collisional-DM model is that it can describe both DM and DE in terms of a single component. In other words, a cosmological model filled with self-interacting DM could be a relatively inexpensive solution to the DE problem. Accordingly, several ways of accommodating both the DM and the DE into a unified theoretical framework have been considered (see, e.g., Zimdahl et al. 2001; Bilić et al. 2002; Balakin et al. 2003; Gondolo \& Freese 2003; Makler et al. 2003; Scherrer 2004; Ren \& Meng 2006; Meng et al. 2007; Lima et al. 2008, 2010, 2012; Basilakos \& Plionis 2009, 2010; Dutta \& Scherrer 2010; Xu et al. 2012), although they have not always been without dispute (see, e.g., Sandvik et al. 2004).

In this context, in a recent work by Kleidis \& Spyrou (2011), it was suggested that the self-interacting DM could phenomenologically attribute to the Universe matter content some sort of fluidlike properties, and consequently lead to a conventional approach to the DE concept. Indeed, the main problem of the current cosmological picture is that the Universe must contain an amount of energy, which is considerably larger than that equivalent to the total rest mass of its matter content. However, if the DM constituents collided with each other frequently enough, enabling their (kinetic) energy to be redistributed, i.e., if the DM itself possessed some sort of thermodynamic properties, then a conventional extra-energy component might be present in the 
Universe, given by the energy of the internal motions of the collisional-DM fluid.

The difference between such a model and those of the classical Friedmann-Robertson-Walker (FRW) cosmology used so far, is that, in this case, the fundamental units of the Universe matter content are the volume elements of the collisional-DM fluid. Hence, together with all the other physical characteristics, the energy of this fluid's internal motions is also taken into account as a source of the universal gravitational field, thus resulting in a self-consistent, conventional approach to the DE concept. Nevertheless, according to Kleidis \& Spyrou (2011), a model in which the DE is attributed to the isothermal flows of a collisional-DM fluid, although it is intriguing, it has two delicate points: not only it is decelerating, but it is also incompatible with the observational data currently available, unless the matter content of the dark sector consists of HDM.

In an effort to confront these issues, we note that in realistic cosmology the polytropic motion of the cosmic fluid's volume elements is much more physically relevant than the corresponding isothermal flow (see, e.g., Christensen-Dalsgard 2008, pp. 64-69). In particular, polytropic processes in a DM fluid have been most successfully used in modeling dark galactic haloes, thus significantly improving the velocity dispersion profiles of galaxies (Bharadwaj \& Kar 2003; Nunez et al. 2006; Zavala et al. 2006; Böhmer \& Harko 2007; Saxton \& Wu 2008; Su \& Chen 2009; Saxton \& Ferreras 2010). Clearly, we cannot help but wonder: What would be the impact of such an assumption on cosmological scale?

Polytropic (DM) cosmological models were first encountered as natural candidates for Cardassian Cosmology models (see, e.g., Freese \& Lewis 2002; Gondolo \& Freese 2003; Wang et al. 2003; Freese 2005). They have also been used as phenomenological models of DE (see, e.g., Nojiri et al. 2005; Stefancić 2005; Mukhopadhyay et al. 2008), especially in an effort to establish an interaction between the exotic DE fluid and its conventional (DM) counterpart (see, e.g., Karami et al. 2009; Karami \& Abdolmaleki 2010a,b, 2012; Malekjani et al. 2011; Chavanis 2014, 2012a,b; Karami \& Khaledian 2012; Asadzadeh et al. 2014).

Our approach, however, is totally different and, in the reasoning of Occam's razor, rather preferable, since it does not involve any DE at all. Instead, we examine the evolution and dynamical properties of a cosmological model driven by a gravitating fluid with thermodynamical content, consisting of DM (dominant) and baryonic matter (subdominant). The fundamental matter constituents of this model are the volume elements of the DM fluid, which perform polytropic flows. As a consequence, now, we also take the energy of this fluid's internal motions into account as a source of the universal gravitational field. This form of energy can compensate for the extra energy, needed to compromise spatial flatness, namely, to justify that, today, the total energy density parameter is exactly unity. The polytropic model depends on only one free parameter, the corresponding exponent, $\Gamma$. We find this model particularly interesting, because for $\Gamma \leq 0.541$, the conventional pressure becomes negative enough in the sense that the Universe accelerates its expansion at cosmological redshifts below a transition value. In fact, several physical reasons, e.g., the cosmological requirement for CDM and a positive velocity-of-sound square, may lead to successive constraints on $\Gamma$, the value of which is eventually settled down to the range $-0.089<\Gamma \leq 0$. The polytropic-DM cosmological model that we propose, does not suffer either from the age problem or from the coincidence problem. At the same time, this model reproduces to high accuracy the distance measurements performed with the aid of the SNe Ia standard candles, without the need for any exotic DE or the cosmological constant. In this context, the value of the CMB-shift parameter in the $\Lambda \mathrm{CDM}$ limit of our model reproduces to high accuracy the corresponding result obtained by fitting the observational (CMB) data to the standard $\Lambda \mathrm{CDM}$ model. Finally, the polytropic-DM model most natutally interprets not only when, but also why the Universe transits from deceleration to acceleration, thus arising as a mighty contestant for a DE model. However, we need to stress that it is not yet clear what type of micro-physics would produce the postulated polytropic behavior; hence, our model is to be seen as an effective (phenomenological) approach of an elementary physics scenario yet to be discovered (in connection, see, e.g., Gondolo \& Freese 2003; Arkani-Hamed et al. 2009; Van den Aarssen et al. 2012).

This paper is organized as follows. In Sect. 2, we summarize the thermodynamical aspect of polytropic processes in an expanding Universe. Accordingly, in Sect. 3, we explore the dynamical characteristics of a spatially-flat cosmological model in which, in principle, there is no DE at all. The evolution of this model is driven by an ideal fluid, consisting of thermodynamically involved DM, the volume elements of which perform polytropic flows. The corresponding results suggest that, in the context of the polytropic treatment, (i) the extra (dark) energy (needed to compromise spatial flatness) can be compensated by the energy of the internal motions of the DM fluid; (ii) the Universe does not suffer either from the age or the coincidence problems; and (iii) such a cosmological model most naturally accelerates its expansion at redshifts lower than a transition value, which depends only on the polytropic exponent, $\Gamma$. We then demonstrate in Sect. 4 that in a polytropic (DM) Universe the theoretically-derived distance modulus fits to high accuracy the Hubble diagram of an extended sample of SNe Ia standard candles, thus emerging as a mighty contestant for a (conventional) DE model. Finally, in Sect. 5, we provide a clear physical interpretation of why and when the Universe transits from deceleration to acceleration, and we conclude in Sect. 6.

\section{Thermodynamics of polytropic processes}

A polytropic change is a reversible process along which the specific heat of a thermodynamical system,

$C=\frac{\mathrm{d} Q}{\mathrm{~d} T}$

varies in a prescribed manner (see, e.g., Horedt 2004, p. 2). An important special case is when $C$ is constant. In this case, along with the fundamental equation of state,

$p \propto \rho T$,

which relates pressure $(p)$ to rest-mass density $(\rho)$ and absolute temperature $(T)$ in a perfect fluid, there is also a second equation, namely $C=$ const., and the polytropic thermodynamical system is left with only one independent state-variable; in our case, this will be the rest-mass density, i.e., the part that is equivalent to the energy density $\rho c^{2}$, which remains unaffected by the internal motions of the cosmic fluid (barotropic flow). Accordingly, Eq. (2) is decomposed to

$p=p_{0}\left(\frac{\rho}{\rho_{0}}\right)^{\Gamma}$

and

$T=T_{0}\left(\frac{\rho}{\rho_{0}}\right)^{\Gamma-1}$ 
Table 1. Thermodynamic processes in an expanding Universe, along with the corresponding values of polytropic exponent, $\Gamma$, and specific heat, $C$.

\begin{tabular}{lll}
\hline \hline Value of $\Gamma$ & Value of $C$ & Process in an expanding Universe \\
\hline$\Gamma \rightarrow-\infty$ & $C \rightarrow C_{\mathrm{V}_{+}}$ & isochoric limit \\
$\Gamma<0$ & $C_{\mathrm{V}}<C<C_{\mathrm{P}}$ & super-isobaric (see, e.g., Kamiuto 2008) \\
$\Gamma=0$ & $C=C_{\mathrm{P}}$ & isobaric - equivalent to $\Lambda$ CDM model (cf. Eq. (64), below) \\
$0<\Gamma<1$ & $C>C_{\mathrm{P}}$ & sub-isobaric \\
$\Gamma \rightarrow 1_{-}$ & $C \rightarrow+\infty$ & isothermal limit $-p<0$ (cf. Eq. (45), below) \\
$\Gamma \rightarrow 1_{+}$ & $C \rightarrow-\infty$ & isothermal limit $-p>0$ (cf. Eq. (45), below) \\
$1<\Gamma<\gamma$ & $C<0$ & quasi-adiabatic \\
$\Gamma=\gamma$ & $C=0$ & adiabatic \\
$\gamma<\Gamma$ & $0<C<C_{\mathrm{V}}$ & not physically-acceptable (cf. Eq. (13), below) \\
$\Gamma \rightarrow+\infty$ & $C \rightarrow C_{\mathrm{V}_{-}}$ & not physically-acceptable (cf. Eq. (13), below) \\
\hline
\end{tabular}

(see, e.g., Chandrasekhar 1939, p. 85; Horedt 2004, p. 9), where $p_{0}, \rho_{0}$, and $T_{0}$ denote the present-time values of pressure, restmass density, and temperature, respectively, and $\Gamma$ is the polytropic exponent, defined as

$\Gamma=\frac{C_{\mathrm{P}}-C}{C_{\mathrm{V}}-C}$

(see, e.g., Chandrasekhar 1939, p. 86; Horedt 2004, p. 5) where $C_{\mathrm{P}}\left(C_{\mathrm{V}}\right)$ is the specific heat at constant pressure (volume).

For $C=0$, the polytropic process is reduced to an adiabatic process $(\mathrm{d} Q=0)$, while, for $C \rightarrow \pm \infty$, it results in an isothermal process $(\mathrm{d} T=0)$. On the other hand, for $C=C_{\mathrm{P}}$, the polytropic becomes an isobaric process ( $p=$ const.), and, for $C \rightarrow C_{\mathrm{V}}$, it yields an isochoric process, although in an expanding Universe this kind of process is possible only as a limiting case (static Universe). Clearly, a polytropic change can be considered as a general treatment, including many thermodynamical processes, as well as their transitional phases, in a single formula. Although $C$ is the real, physical quantity, it is useful to parametrize it in terms of $\Gamma$, and hence address the impact of the polytropic exponent to each and everyone of these processes. Accordingly, we express Eq. (5) in the form

$C=\left[1-\left(\frac{\gamma-1}{\Gamma-1}\right)\right] C_{\mathrm{V}}$

where

$\gamma=\frac{C_{\mathrm{P}}}{C_{\mathrm{V}}}$

is the adiabatic exponent. Indicative only, for $\gamma=\frac{5}{3}$, the behavior of $C$ as a function of $\Gamma$, is presented in Fig. 1. The various processes encountered in Fig. 1 are summarized in Table 1.

As we observe, the concept of polytropic process is of great importance (at least) from a methodological viewpoint, since it correlates all other known thermodynamical processes.

However, not all of these processes are physically acceptable in an expanding Universe. For example, in a cosmological model with matter content in the form of perfect fluid, processes 9 and 10 of Table 1 are excluded, because of the second law of thermodynamics. In fact, according to this law, the entropy, $\mathcal{S}$, of a reversible process, obeying

$\mathrm{d} \mathcal{S}=\frac{\mathrm{d} Q}{T}$,

is a never-diminishing function, i.e.,

$\Delta \mathcal{S}=\mathcal{S}_{\text {final }}-\mathcal{S}_{\text {initial }} \geq 0$,

(9) $\Gamma \leq \gamma$

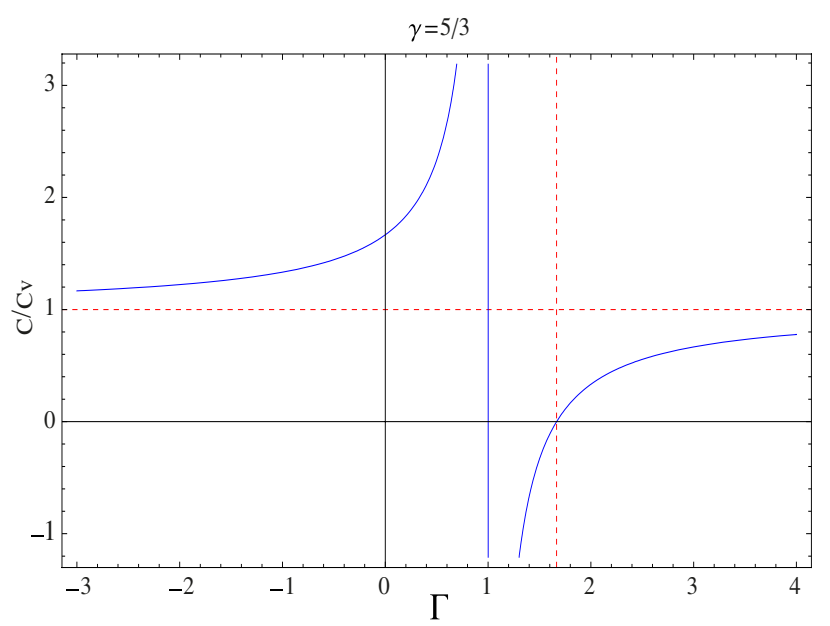

Fig. 1. Behavior of the specific heat, $C$ (in units of $C_{\mathrm{V}}$ ), as a function of the polytropic exponent, $\Gamma$ (blue solid curve), for $\gamma=\frac{5}{3}$ (vertical red dashed line).

where the equality holds only for adiabatic processes. To determine the variation of entropy, along the polytropic transition of a perfect (cosmic) fluid from an initial state to a final state (the present epoch), we insert Eq. (1) into Eq. (8) and integrate, to obtain

$\mathcal{S}(t)-\mathcal{S}_{0}=C \ln \left[\frac{T(t)}{T_{0}}\right]$,

where $\mathcal{S}(t)$ is the entropy of the fluid at some $t \leq t_{0}$ and $\mathcal{S}_{0}$ is the corresponding value at the present epoch $\left(t_{0}\right)$. In view of Eq. (4), Eq. (10) yields

$\mathcal{S}(t)=\mathcal{S}_{0}+(\Gamma-1) C \ln \left[\frac{\rho(t)}{\rho_{0}}\right]$

and, by virtue of Eq. (6), it results in

$\mathcal{S}(t)=\mathcal{S}_{0}+(\Gamma-\gamma) C_{\mathrm{V}} \ln \left[\frac{\rho(t)}{\rho_{0}}\right]$.

In an expanding Universe, with matter in the form of a calorically-perfect fluid, $C_{\mathrm{V}}=(\partial \mathcal{U} / \partial T)_{\mathrm{V}}=$ const. $>0$ (see, e.g., Christians 2012), where $\mathcal{U}$ is the energy of this fluid's internal motions (per unit rest-mass). Furthermore, admitting that each volume element of the polytropic fluid is a closed thermodynamical system, total rest-mass (i.e., particles' number) consrvation implies that, for every $t \leq t_{0}, \rho(t) \geq \rho_{0}$. As a consequence, in view of Eq. (12), the second law of thermodynamics, given by Eq. (9), suggests that 
and, hence, in a realistic polytropic cosmological model, the whole range of values $\Gamma>\gamma$ is excluded. According to the condition given by Eq. (13), in an expanding Universe filled with polytropic perfect fluid, there are only two physically acceptable domains of values of the polytropic exponent, namely,

$$
\begin{gathered}
-\infty<\Gamma<1 \Leftrightarrow C>0 \Leftrightarrow d T>0 \text { and } \\
1<\Gamma \leq \gamma \Leftrightarrow C \leq 0 \Leftrightarrow d T<0,
\end{gathered}
$$

separated by the isothermal $(\Gamma=1)$ barrier, for which $d T=0$. Along with the expansion toward the present epoch, the temperature of a polytropic perfect fluid with $\Gamma<1$ increases $(\mathrm{d} T>0)$ and, therefore, so does its internal energy.

On the other hand, the work (per unit mass) done by the pressure along the polytropic expansion of the cosmic fluid from an initial state (at which, $\rho=\rho(t)$ ) to a final state (the present epoch, at which $\left.\rho=\rho_{0}<\rho(t)\right)$, is given by

$W_{\rho \rightarrow \rho_{0}}=\int_{\rho}^{\rho_{0}} p \mathrm{~d} V=\int_{\rho}^{\rho_{0}} p \mathrm{~d}\left(\frac{1}{\rho}\right)$,

which, by virtue of Eq. (3) (for $\Gamma \neq 1$ ), yields

$W_{\rho \rightarrow \rho_{0}}=\frac{1}{\Gamma-1} \frac{p_{0}}{\rho_{0}}\left[\left(\frac{\rho}{\rho_{0}}\right)^{\Gamma-1}-1\right]$.

As we shall clarify below (see e.g., Eq. (45)), the present-time value of the (conventional) pressure along a polytropic process in an expanding spatially-flat FRW model, is given by

$p_{0}=\rho_{0} c^{2}(\Gamma-1) \frac{1-\Omega_{\mathrm{M}}}{\Omega_{\mathrm{M}}}$.

Hence, Eq. (17) results in

$W_{\rho \rightarrow \rho_{0}}=\frac{1-\Omega_{\mathrm{M}}}{\Omega_{\mathrm{M}}} c^{2}\left[\left(\frac{\rho}{\rho_{0}}\right)^{\Gamma-1}-1\right]$.

In this case, for $\Gamma<1$ (i.e., $\Gamma-1=-|1-\Gamma|$ ), Eq. (19) is written in the form

$W_{\rho \rightarrow \rho_{0}}^{\Gamma<1}=-\frac{1-\Omega_{\mathrm{M}}}{\Omega_{\mathrm{M}}} c^{2}\left[1-\left(\frac{\rho_{0}}{\rho}\right)^{|1-\Gamma|}\right]<0$.

Since $\Omega_{M}<1$, Eq. (20) implies that, for $\Gamma<1$, the work done by the pressure (during the cosmic expansion of a polytropic perfect fluid) is negative, i.e., it is returned to the cosmic fluid itself.

Eventually, in a polytropic cosmological model, the first law of thermodynamics is given by

$\mathrm{d} \mathcal{U}+p \mathrm{~d}\left(\frac{1}{\rho}\right)=C \mathrm{~d} T$

(see, e.g., Fock 1959, p. 83). In view of Eqs. (14) and (20), Eq. (21) suggests that along a polytropic transition with $\Gamma<1$, the internal energy of the cosmic fluid increases $(\mathrm{d} \mathcal{U}>0)$, as a result of both $\mathrm{d} T>0$ and $\mathrm{d} W=p \mathrm{~d}\left(\frac{1}{\rho}\right)<0$. Hence, there might be a point, at which, the thermodynamical-energy density, attributed to the internal motions of a polytropic cosmic fluid with $\Gamma<1$, dominates over the corresponding rest-mass quantity (i.e., $\rho \mathcal{U}>\rho c^{2}$ ), thus arising as a mighty contestant for the compensation of the extra (dark) energy amount, needed to flatten the Universe. In this context, a polytropic cosmological model could be a viable (and conventional) alternative to the $\mathrm{DE}$ concept, and, for this reason, in the sections that follow, we shall scrutinize this model. Nevertheless, it is not yet clear what kind of microphysics would produce the postulated polytropic behavior, and, therefore, our model is to be seen as an effective approach of an elementary physics scenario yet to be discovered.

\section{Polytropic processes in a cosmological (DM) fluid}

\subsection{The Universe matter-energy content}

The beginning of the 21 st century has been one of the most exciting epochs for cosmology as a science. According to the various CMB-oriented observational data, which became public at that time (see, e.g., de Bernardis et al. 2000; Jaffe et al. 2001; Padin et al. 2001; Stompor et al. 2001; Netterfield et al. 2002), the Universe can be adequately described by a spatially-flat RW model

$\mathrm{d} s^{2}=c^{2} \mathrm{~d} t^{2}-S^{2}(t)\left(\mathrm{d} x^{2}+\mathrm{d} y^{2}+\mathrm{d} z^{2}\right)$,

where $S(t)$ is the cosmic scale factor as a function of time. In such a model, the value of the Hubble parameter, $H(t)$, at the present epoch, is, by definition, given by

$H^{2}\left(t_{0}\right)=\frac{8 \pi G}{3} \rho_{\mathrm{c}}=H_{0}^{2}$

(see, e.g., Peacock 1999, p. 77). In the context of general relativity (GR), the evolution of this model also depends on the source that drives the universal gravitational field, i.e., its matter-energy content.

In specifying the Universe matter-energy content, we assume that, in principle, there is no DE at all. Instead, we admit that the $\mathrm{DM}$ along with the small, baryonic contamination possesses fluidlike properties, in the sense that the collisions of the WIMPs maintain a tight coupling between them and, hence, their (kinetic) energy can be redistributed. In this case, the fundamental units of the Universe matter-energy content are the volume elements of the collisional-DM fluid (elements of fluid, each always consisting always of the same number of particles), which, as we assume, perform polytropic flows.

In view of Bianchi identities, the motion of the volume elements in the interior of a gravitating continuous medium is governed by the equations

$T_{; v}^{\mu v}=0$

where Greek indices refer to the four-dimensional spacetime (in connection, Latin indices refer to the three-dimensional spatial slices), the semicolon denotes covariant derivative, and $T^{\mu v}$ is the energy-momentum tensor of the Universe matter content, i.e., basically (but not solely) of the polytropic-DM fluid. Confining ourselves to the particular case of a perfect fluid, $T^{\mu v}$ takes on the standard form

$T^{\mu v}=(\varepsilon+p) u^{\mu} u^{v}-p g^{\mu v}$,

where $u^{\mu}=\mathrm{d} x^{\mu} / \mathrm{d} s$ is the four-velocity $\left(u_{\mu} u^{\mu}=1\right)$ at the position of a fluid's volume element, $g^{\mu v}$ are the contravariant components of the Universe metric tensor, and $\varepsilon$ is this fluid's total energy density. Provided that internal structure is evident, in an ideal equilibrium state, $\varepsilon$ can be decomposed according to

$\varepsilon=w(\rho, T)+\rho \mathcal{U}(T)$

(for a detailed analysis see, e.g., Fock 1959, pp. 81-83 and 91-94), where $\rho \mathcal{U}$ is the energy density associated with this fluid's thermodynamical content and $w$ denotes every other form of energy (density) involved. To determine $\mathcal{U}$ and $w$ (and, through them, $\varepsilon$, as well), we address thermodynamics and relativity, respectively. 
The first law of thermodynamics:

By virtue of Eqs. (3) and (4), the first law of thermodynamics (given by Eq. (21)) for polytropic flows yields

$\mathcal{U}=\mathcal{U}_{0}\left(\frac{\rho}{\rho_{0}}\right)^{\Gamma-1}$

i.e., $\mathcal{U} \sim T$ (cf. Eq. (4)), where

$\mathcal{U}_{0}=C T_{0}+\frac{1}{\Gamma-1} \frac{p_{0}}{\rho_{0}}$

denotes the present-time value of the cosmic fluid's internal energy. Accordingly, the total-energy density of the Universe matter-energy content is written in the form

$\varepsilon=w+\rho_{0} \mathcal{U}_{0}\left(\frac{\rho}{\rho_{0}}\right)^{\Gamma}$

The continuity equation of GR:

On the other hand, in terms of the metric tensor given by Eq. (22), the conservation law $T_{; v}^{0 v}=0$ reads

$\dot{\varepsilon}+3 \frac{\dot{S}}{S}(\varepsilon+p)=0$

where the dot denotes differentiation with respect to cosmic time, $t$. Upon consideration of Eqs. (3) and (27)-(29), Eq. (30) results in

$\Gamma \mathcal{U}_{0}\left(\dot{\rho}+3 \frac{\dot{S}}{S} \rho\right)+\dot{w}+3 \frac{\dot{S}}{S} w-3(\Gamma-1) \rho_{0} C T_{0} \frac{\dot{S}}{S}\left(\frac{\rho}{\rho_{0}}\right)^{\Gamma}=0$

At this point, we recall that, by definition, each volume element of the polytropic-DM fluid corresponds to a closed thermodynamical system, i.e., its particles' number is conserved. Consequently,

$\dot{\rho}+3 \frac{\dot{S}}{S} \rho=0$,

and, hence,

$\rho=\rho_{0}\left(\frac{S_{0}}{S}\right)^{3}$,

where $S_{0}$ is the value of $S(t)$ at the present epoch. In fact, Eq. (33) represents the conservation of the total rest mass in a FRW cosmological model. In view of Eqs. (32) and (33), Eq. (31) is written in the form

$\dot{w}+3 \frac{\dot{S}}{S} w-3(\Gamma-1) \rho_{0} C T_{0} \frac{\dot{S}}{S}\left(\frac{S_{0}}{S}\right)^{3 \Gamma}=0$,

i.e., as a linear differential equation of the first order to $w$, the solution of which reads

$w=\frac{A}{S^{3}}-\rho_{0} C T_{0}\left(\frac{S_{0}}{S}\right)^{3 \Gamma}$,

where $A$ is an integration constant. Taking into account Eq. (33), the dependence of the first term on the scale factor leads us to identify $A$ with the present time value of the energy density corresponding to the total rest mass, namely,

$A=\rho_{0} c^{2} S_{0}^{3}$.
Accordingly, Eq. (35) takes on its final form, as

$w=\rho_{0} c^{2}\left(\frac{S_{0}}{S}\right)^{3}-\rho_{0} C T_{0}\left(\frac{S_{0}}{S}\right)^{3 \Gamma}$,

that is,

$w=\rho c^{2}-\rho C T$,

in which, the second term represents the heat per unit (of specific) volume, $Q /\left(\frac{1}{\rho}\right)$, entering into the thermodynamical system. Eventually, by virtue of Eq. (37), Eq. (29) is written in the form

$\varepsilon=\rho_{0} c^{2}\left(\frac{S_{0}}{S}\right)^{3}+\frac{p_{0}}{\Gamma-1}\left(\frac{S_{0}}{S}\right)^{3 \Gamma}=\rho c^{2}+\frac{p}{\Gamma-1}$,

where we have also used Eq. (33). Upon consideration of Eq. (39), the dynamical evolution of the cosmological model given by Eq. (22), is governed by the Friedmann equation (with $\Lambda=0$ ) of the classical FRW Cosmology

$H^{2}=\frac{8 \pi G}{3 c^{2}} \varepsilon$,

where

$H=\frac{\dot{S}}{S}$

is the Hubble parameter as a function of the scale factor. However, there is an essential difference between this model and the rest of the classical FRW cosmologies: in this case, the basic matter constituents are the volume elements of a polytropic-DM fluid, i.e., they possess some sort of internal structure, and, therefore, thermodynamical content. As a consequence, the functional form of $\varepsilon$ in Eq. (40) is no longer given by $\rho c^{2}$ alone, but by Eq. (39) (in connection, see Narlikar 1983, pp. 61, 62), and the Friedmann equation reads

$H^{2}=\frac{8 \pi G}{3} \rho_{0}\left(\frac{S_{0}}{S}\right)^{3}\left[1+\frac{1}{\Gamma-1} \frac{p_{0}}{\rho_{0} c^{2}}\left(\frac{S_{0}}{S}\right)^{3(\Gamma-1)}\right]$,

which, in view of Eq. (23), results in

$\left(\frac{H}{H_{0}}\right)^{2}=\Omega_{\mathrm{M}}\left(\frac{S_{0}}{S}\right)^{3}\left[1+\frac{1}{\Gamma-1} \frac{p_{0}}{\rho_{0} c^{2}}\left(\frac{S_{0}}{S}\right)^{3(\Gamma-1)}\right]$.

At the present epoch, when $S=S_{0}$ and $H=H_{0}$, Eq. (43) is reduced to

$\Omega_{\mathrm{M}}\left(1+\frac{1}{\Gamma-1} \frac{p_{0}}{\rho_{0} c^{2}}\right)=1$,

from which, the present time value of the pressure arises, as

$p_{0}=\rho_{0} c^{2}(\Gamma-1) \frac{1-\Omega_{\mathrm{M}}}{\Omega_{\mathrm{M}}}$,

and the Friedmann Eq. (43) is written in the form

$\left(\frac{H}{H_{0}}\right)^{2}=\left(\frac{S_{0}}{S}\right)^{3}\left[\Omega_{\mathrm{M}}+\left(1-\Omega_{\mathrm{M}}\right)\left(\frac{S}{S_{0}}\right)^{3(1-\Gamma)}\right]$.

For $\Gamma<1$, i.e., $C>C_{\mathrm{V}}$ (see, e.g., Fig. 1), Eq. (45) suggests that the pressure given by Eq. (3), referring to a gravitating perfect fluid that consists of polytropic DM, is negative. In this case, the quantity $\varepsilon+3 p$ may also become negative (at some value of $S \leq S_{0}$ ), something that leads to $\ddot{S}>0$ (see, e.g., 
Linder 2008; Caldwell \& Kamionkowski 2009). In other words, a cosmological model filled with a $(\Gamma<1)$ polytropic fluid, may accelerate its expansion (see Sect. 5.3, below).

On the contrary, any cosmological model filled with matter in the form of a polytropic perfect fluid with $\Gamma>1$ (i.e., of positive pressure), is ever-decelerating. Indeed, for $\Gamma \rightarrow 1_{+}$, i.e., $\Gamma=1+\epsilon$ with $\epsilon \rightarrow 0$, Eq. (46) results in

$\left(\frac{H}{H_{0}}\right)^{2}=\left(\frac{S_{0}}{S}\right)^{3}\left[1+3 \epsilon\left(1-\Omega_{\mathrm{M}}\right) \ln \left(\frac{S_{0}}{S}\right)+O\left(\epsilon^{2}\right)\right]$,

which, to linear terms in $\epsilon$, is of the same functional form as Eq. (24) of Kleidis \& Spyrou (2011), thus resulting in an everdecelerating Universe.

Furthermore, by virtue of Eq. (45), Eq. (39), for every value of $\Gamma$, is written in the form

$\varepsilon=\rho_{0} c^{2}\left[\left(\frac{S_{0}}{S}\right)^{3}+\frac{1-\Omega_{\mathrm{M}}}{\Omega_{\mathrm{M}}}\left(\frac{S_{0}}{S}\right)^{3 \Gamma}\right]$,

or

$\varepsilon=\rho_{\mathrm{c}} c^{2}\left[\Omega_{\mathrm{M}}\left(\frac{S_{0}}{S}\right)^{3}+\left(1-\Omega_{\mathrm{M}}\right)\left(\frac{S_{0}}{S}\right)^{3 \Gamma}\right]>0$.

Accordingly, in a cosmological model in which polytropic processes are dominant, the present time (i.e., when $S=S_{0}$ ) value of the total energy density, $\Omega_{0}$, equals to unity, i.e.,

$\Omega_{0}=\frac{\varepsilon_{0}}{\varepsilon_{\mathrm{c}}}=\frac{\rho_{\mathrm{c}} c^{2}}{\rho_{\mathrm{c}} c^{2}}\left[\Omega_{\mathrm{M}}+\left(1-\Omega_{\mathrm{M}}\right)\right]=1$.

In view of Eqs. (49) and (50), the extra (dark) energy, needed to compromise spatial flatness of the cosmological model given by Eq. (22), can be provided by the energy of the internal motions of a collisional-DM fluid, the volume elements of which perform polytropic flows. Hence, a Universe with matter content in the form of a polytropic-DM fluid with $\Gamma<1$, might be a relatively inexpensive solution to the whole DE concept, in the sense that it can address both the extra energy needed for spatial flatness and the subsequent accelerated expansion in one single model. For this reason, in what follows we shall focus on a cosmological model filled with a polytropic (DM) perfect fluid with $\Gamma<1$.

In such a model, by virtue of Eq. (48), we can identify the rest-mass energy density, $\varepsilon_{\text {mat }}=\rho c^{2}$, and the extra (dark) energy density, $\varepsilon_{\text {int }}=\varepsilon-\varepsilon_{\text {mat }}$, of the Universe total matter-energy content and express these quantities as functions of the cosmological redshift parameter,

$z+1=\frac{S_{0}}{S}$

yielding

$\frac{\varepsilon_{\text {int }}}{\varepsilon_{\text {mat }}}=\frac{1-\Omega_{\mathrm{M}}}{\Omega_{\mathrm{M}}} \frac{1}{(1+z)^{3(1-\Gamma)}}$.

At the present epoch $(z=0)$, and for every value of $\Gamma$, Eq. (52) results in

$\left.\frac{\varepsilon_{\text {int }}}{\varepsilon_{\text {mat }}}\right|_{0}=\frac{1-\Omega_{\mathrm{M}}}{\Omega_{\mathrm{M}}}$,

as it should in a spatially-flat Universe.

Notice that, for $\Omega_{\mathrm{M}}=0.274$ (Komatsu et al. 2011), Eq. (45) suggests that, today, $p_{0}=-2.650(1-\Gamma) \rho_{0} c^{2}$. This result could create the assumption that the polytropic cosmological model is nothing but a phantom Universe where $p_{0}<-\varepsilon_{0}$. However, we need to stress that by virtue of Eq. (48), in this cosmological model, the total energy density at the present epoch is not given by $\rho_{0} c^{2}$, but by $\varepsilon_{0}=\Omega_{\mathrm{M}}^{-1} \rho_{0} c^{2}$. Accordingly, Eq. (45) results in

$p_{0}=-(1-\Gamma)\left(1-\Omega_{\mathrm{M}}\right) \varepsilon_{0}$.

In this case, as long as

$(1-\Gamma)\left(1-\Omega_{\mathrm{M}}\right)<1 \Leftrightarrow \Gamma>-\frac{\Omega_{\mathrm{M}}}{1-\Omega_{\mathrm{M}}} \cong-0.377$,

we obtain

$p_{0}>-\varepsilon_{0}$

and, therefore, the polytropic-DM model no longer belongs to the realm of phantom cosmology. Indeed, as we shall demonstrate later on, Eq. (55) is valid in a cosmological model in which the accelerated expansion takes place at a lower rate than de Sitter expansion (cf. also Eq. (75)).

\subsection{The Universe scale factor and the cosmic time}

In a cosmological model filled with a polytropic perfect fluid, Eq. (46) yields

$\left[\frac{\mathrm{d}}{\mathrm{d} t}\left(\frac{S}{S_{0}}\right)^{3 / 2}\right]^{2}=\frac{1}{t_{\mathrm{EdS}}^{2}}\left\{\Omega_{\mathrm{M}}+\left(1-\Omega_{\mathrm{M}}\right)\left[\left(\frac{S}{S_{0}}\right)^{3 / 2}\right]^{2(1-\Gamma)}\right\}$,

where

$t_{\text {EdS }}=\frac{2}{3 H_{0}}$

is the age of the Universe of the Einstein-de Sitter (EdS) model (dust Universe). To solve Eq. (57), we set

$0 \leq \chi=\left(\frac{S}{S_{0}}\right)^{3 / 2} \leq 1$

In accordance, Eq. (57) results in

$\int_{0}^{\chi} \frac{d \chi}{\sqrt{\Omega_{\mathrm{M}}+\left(1-\Omega_{\mathrm{M}}\right) \chi^{2(1-\Gamma)}}}=\frac{t}{t_{\mathrm{EdS}}}$.

Equation (60) can be solved explicitly in terms of hypergeometric functions, ${ }_{2} F_{1}(a, b ; c ; x)$, of a complex variable, $x$ (see, e.g., Gradshteyn \& Ryzhik 2007 (7th edn.), pp. 1005-1008), as follows

$$
\begin{aligned}
& \left(\frac{S}{S_{0}}\right)^{\frac{3}{2}} \times{ }_{2} F_{1}\left(\frac{1}{2(1-\Gamma)}, \frac{1}{2} ; \frac{3-2 \Gamma}{2(1-\Gamma)} ;-\left(\frac{1-\Omega_{\mathrm{M}}}{\Omega_{\mathrm{M}}}\right)\left[\frac{S}{S_{0}}\right]^{3(1-\Gamma)}\right)= \\
& \sqrt{\Omega_{\mathrm{M}}}\left(\frac{t}{t_{\mathrm{EdS}}}\right),
\end{aligned}
$$

which, for $\Omega_{\mathrm{M}}=1$, yields $S=S_{0}\left(\frac{t}{t_{\mathrm{EdS}}}\right)^{2 / 3}$, i.e., the EdS model, as it should. Since $a+b=\frac{1}{2(1-\Gamma)}+\frac{1}{2}<\frac{3-2 \Gamma}{2(1-\Gamma)}=c$, the hypergeometric series involved in Eq. (61), converges absolutely within the unit circle $\left|\frac{S}{S_{0}}\right| \leq 1$, for every value of $\Gamma<1$ (see, e.g., Abramowitz \& Stegun 1970, p. 556). In the isobaric $\Gamma=0$ case, Eq. (61) is reduced to

$$
\left(\frac{S}{S_{0}}\right)^{\frac{3}{2}}{ }_{2} F_{1}\left(\frac{1}{2}, \frac{1}{2} ; \frac{3}{2} ;-\left(\frac{1-\Omega_{\mathrm{M}}}{\Omega_{\mathrm{M}}}\right)\left[\frac{S}{S_{0}}\right]^{3}\right)=\sqrt{\Omega_{\mathrm{M}}}\left(\frac{t}{t_{\mathrm{EdS}}}\right),
$$




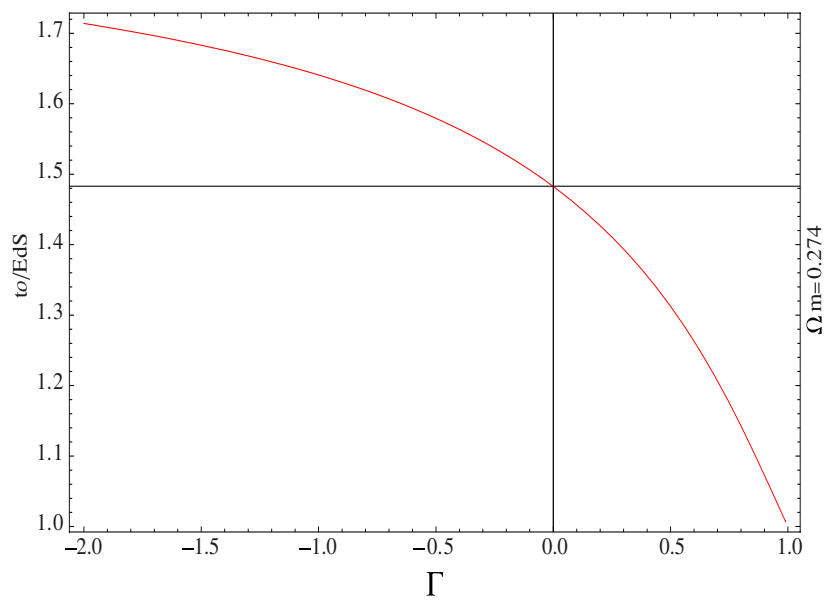

Fig. 2. Age of a polytropic-DM model, $t_{0}$, in units of $t_{\mathrm{EdS}}$, as a function of the polytropic exponent $\Gamma<1$ (red solid line). For every $\Gamma<1$, $t_{0}>t_{\mathrm{EdS}}$, and $t_{0}$ approaches $t_{\mathrm{EdS}}$ only as $\Gamma \rightarrow 1$. The horizontal solid line denotes the age of the Universe, $t_{0}=1.483 t_{\mathrm{Eds}}$, in the $\Lambda \mathrm{CDM}$ limit of the polytropic-DM model (i.e., for $\Gamma=0$ ).

which, upon consideration of the identity

${ }_{2} F_{1}\left(\frac{1}{2}, \frac{1}{2} ; \frac{3}{2} ;-x^{2}\right)=\frac{1}{x} \sinh ^{-1}(x)$

(cf. Abramowitz \& Stegun 1970, Eq. (15.1.7), p. 556; Gradshteyn \& Ryzhik 2007 (7th edn.), Eq. (9.121.28), p. 1007), results in

$S(t)=S_{0}\left(\frac{\Omega_{\mathrm{M}}}{1-\Omega_{\mathrm{M}}}\right)^{1 / 3} \sinh ^{2 / 3}\left(\sqrt{1-\Omega_{\mathrm{M}}} \frac{t}{t_{\mathrm{EdS}}}\right)$,

i.e., in a functional form similar to the corresponding $\Lambda \mathrm{CDM}$ result. Moreover, from Eq. (61) we may determine the age of the Universe, $t_{0}$ (i.e., the time at which $S=S_{0}$ ), in a polytropic-DM model. In units of $t_{\mathrm{EdS}}$, it is given by

$$
\begin{aligned}
\frac{t_{0}}{t_{\mathrm{EdS}}}= & \frac{1}{\sqrt{\Omega_{\mathrm{M}}}} \\
& \times{ }_{2} F_{1}\left(\frac{1}{2(1-\Gamma)}, \frac{1}{2} ; 1+\frac{1}{2(1-\Gamma)} ;-\frac{1-\Omega_{\mathrm{M}}}{\Omega_{\mathrm{M}}}\right),
\end{aligned}
$$

the behavior of which, as a function of the polytropic exponent, $\Gamma<1$, is presented in Fig. 2. For $p=$ const. $=p_{0}$ (i.e., $\Gamma=0$ ), Eq. (65) yields

$t_{0}=t_{\mathrm{EdS}} \frac{1}{\sqrt{1-\Omega_{\mathrm{M}}}} \sinh ^{-1} \sqrt{\frac{1-\Omega_{\mathrm{M}}}{\Omega_{\mathrm{M}}}}$,

which, for $\Omega_{\mathrm{M}}=0.274$ (Komatsu et al. 2011), results in $t_{0}=$ $1.483 t_{\mathrm{EdS}}=13.773$ Gys, i.e., the age of the Universe of the $\Lambda$ CDM model.

Eventually, combining Eqs. (61) and (65), we obtain the equation that governs the time evolution of a polytropic-DM model. It is written in the form

$\left(\frac{S}{S_{0}}\right)^{3 / 2} \times \frac{{ }_{2} F_{1}\left(\frac{1}{2(1-\Gamma)}, \frac{1}{2} ; \frac{3-2 \Gamma}{2(1-\Gamma)} ;-\left(\frac{1-\Omega_{\mathrm{M}}}{\Omega_{\mathrm{M}}}\right)\left[\frac{S}{S_{0}}\right]^{3(1-\Gamma)}\right)}{{ }_{2} F_{1}\left(\frac{1}{2(1-\Gamma)}, \frac{1}{2} ; \frac{3-2 \Gamma}{2(1-\Gamma)} ;-\frac{1-\Omega_{\mathrm{M}}}{\Omega_{\mathrm{M}}}\right)}=\frac{t}{t_{0}} \cdot(67)$

In this case, the time behavior of the cosmic scale factor, for several values of the polytropic exponent $\Gamma<1$, is presented

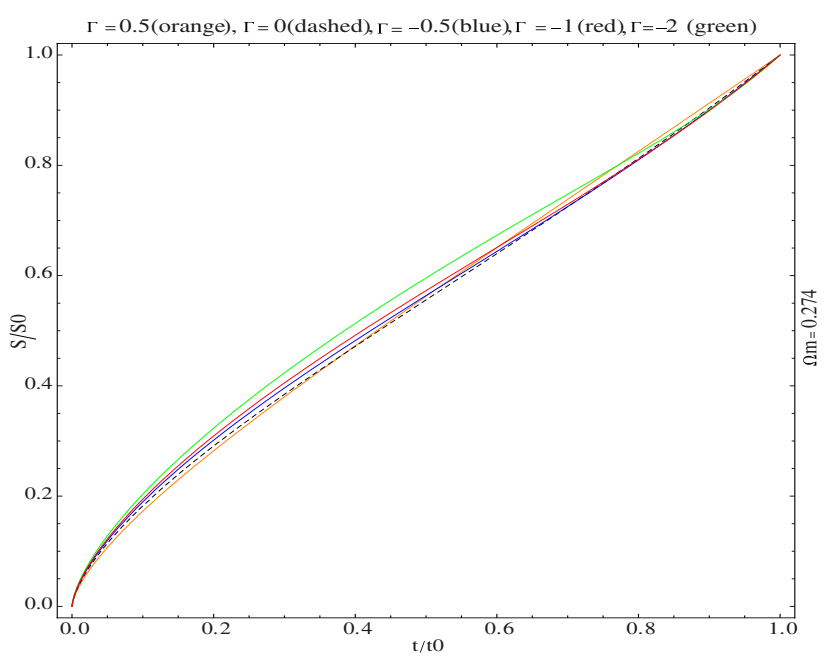

Fig. 3. Scale factor, $S$, of a cosmological model driven by a polytropicDM fluid with $\Omega_{\mathrm{M}}=0.274$ (in units of its present-time value, $S_{0}$ ), as a function of the cosmic time $t$ (in units of $t_{0}$ ), for $\Gamma=0.5$ (orange), $\Gamma=0$ (dashed), $\Gamma=-0.5$ (blue), $\Gamma=-1$ (red), and $\Gamma=-2$ (green). For each of these curves, there is always a value of $t<t_{0}$ above which $S(t)$ becomes concave, i.e., $\ddot{S}>0$, and, hence, the Universe accelerates its expansion.

in Fig. 3. The profiles of the corresponding curves suggest that there is always a value of $t<t_{0}$, above which, the function $S(t)$ becomes concave (i.e., $\ddot{S}>0$ ); in other words, a cosmological model filled with a polytropic (DM) perfect fluid, for $\Gamma<1$, accelerates its expansion. This can be confirmed, if someone calculates the corresponding deceleration parameter, $q$.

\subsection{Deceleration parameter and the subsequent accelerated expansion}

In a cosmological model in which the polytropic flow (with $\Gamma<1$ ) of the cosmic fluid's volume elements is the dominant type of motion, the Hubble parameter (46), in terms of the cosmological redshift parameter, $z$, is written in the form

$H=H_{0}(1+z)^{\frac{3}{2}}\left[\Omega_{\mathrm{M}}+\frac{1-\Omega_{\mathrm{M}}}{(1+z)^{3(1-\Gamma)}}\right]^{1 / 2}$.

Accordingly, the corresponding deceleration parameter, in terms of $H$ and $z$, is given by

$q(z)=\frac{\mathrm{d} H / \mathrm{d} z}{H(z)}(1+z)-1$,

which, upon consideration of Eq. (68), yields

$q(z)=\frac{1}{2}\left[1-\frac{3(1-\Gamma)\left(1-\Omega_{\mathrm{M}}\right)}{\Omega_{\mathrm{M}}(1+z)^{3(1-\Gamma)}+\left(1-\Omega_{\mathrm{M}}\right)}\right]$.

For $z \gg 1$ (i.e., at the distant past), $q \rightarrow \frac{1}{2}$ and the Universe behaves as a matter (dust) dominated (in other words, decelerating) FRW model. On the other hand, for $z=0$ (i.e., at the present epoch), we have

$q_{0}=\frac{1}{2}\left[1-3(1-\Gamma)\left(1-\Omega_{\mathrm{M}}\right)\right]$,

which, depending on $\Gamma$, can be either positive, negative, or zero. In fact, the minus sign on the rhs of Eq. (70) suggests that there 


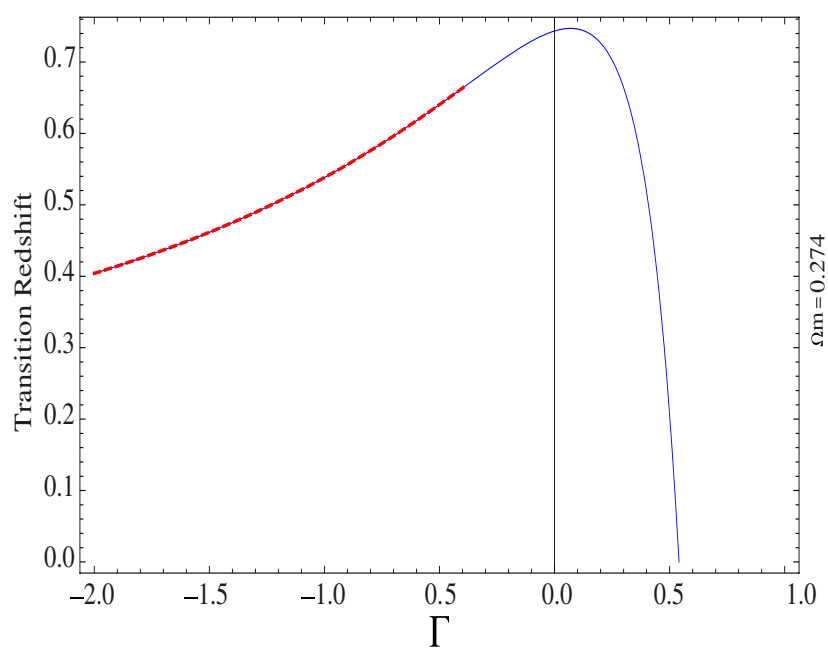

Fig. 4. Transition redshift, $z_{\text {tr }}$, in a polytropic-DM model as a function of the polytropic exponent, $\Gamma$ (blue solid curve). The constraint $z_{\operatorname{tr}} \geq 0$ yields $\Gamma \leq 0.541$, while the condition $q>-1$ results in $\Gamma>-0.377$ below which the Universe enters in the phantom realm (red dashed curve).

is a transition value of $z$, namely, $z_{\mathrm{tr}}$, below which, $q(z)$ does become negative, i.e., the Universe accelerates its expansion. In a polytropic-DM model, $z_{\text {tr }}$ is a function of the polytropic exponent, given by

$z_{\mathrm{tr}}=\left[(2-3 \Gamma) \frac{1-\Omega_{\mathrm{M}}}{\Omega_{\mathrm{M}}}\right]^{\frac{1}{3(1-\Gamma)}}-1$

In view of Eq. (6), in a polytropic fluid with $C>C_{\mathrm{V}}$ (i.e., $\Gamma<1$ ), the condition

$\Gamma=\frac{\gamma-\frac{C}{C_{\mathrm{V}}}}{1-\frac{C}{C_{\mathrm{V}}}}<\frac{2}{3}$

leads to $\gamma-\frac{2}{3}>\frac{1}{3}\left(\frac{C}{C_{\mathrm{V}}}\right)>\frac{1}{3} \Rightarrow \gamma>1$ which is valid anyway. Hence, in what follows we consider $\Gamma<\frac{2}{3}$ (in connection, see also Freese \& Lewis 2002; Gondolo \& Freese 2003). In this context, the condition $z_{\text {tr }} \geq 0$ (equivalently, $q_{0} \leq 0$ ) imposes a further constraint on $\Gamma$ itself, namely,

$\Gamma \leq \frac{1}{3}\left[2-\frac{\Omega_{\mathrm{M}}}{1-\Omega_{\mathrm{M}}}\right]$,

which, for $\Omega_{\mathrm{M}}=0.274$ (Komatsu et al. 2011), yields an upper limit of $\Gamma$, namely, $\Gamma \leq 0.541$. On the other hand, admitting that the accelerating Universe will approach a de Sitter phase only in the distant future, the condition $q_{0}>-1$ (equivalently, $p_{0}>-\varepsilon_{0}$ ) should (also) be imposed, leading to

$\Gamma>-\frac{\Omega_{\mathrm{M}}}{1-\Omega_{\mathrm{M}}} \cong-0.377$,

which may serve as a lower bound of $\Gamma$ (cf. also Eq. (55)). Finally, for $\Gamma=0$, Eq. (72) yields $z_{\text {tr }}=0.744$, which lies within the current range of the corresponding $\Lambda \mathrm{CDM}$ result, namely, $z_{\text {tr }}=0.752 \pm 0.041$ (see, e.g., Suzuki et al. 2012). The behavior of $z_{\mathrm{tr}}$, as a function of $\Gamma \leq 0.541$, is presented in Fig. 4 .

In view of the aforementioned resutls, polytropic acceleration is certainly not a coincidence. In other words, a cosmological model filled with a polytropic (DM) perfect fluid of $-0.377<$ $\Gamma \leq 0.541$, most naturally accelerates its expansion at cosmological redshifts lower than a transition value, given by Eq. (72), without the need for either any exotic DE or the cosmological constant.

The question that arises now is whether or not these results can also be confirmed by observational data of cosmological significance, especially those that led to the assumption of the accelerating expansion in the first place. In fact, as we demonstrate in the next section, a Universe with matter content in the form of a polytropic-DM fluid, can reproduce to high accuracy the observational distribution of the SNe Ia distant indicators.

\section{Accommodating the $\mathrm{SN}$ la observational data}

Nowadays, the most direct and reliable method for observationally determining the recent history of the Universe expansion is to measure the redshift and the apparent luminosity (equivalently, the apparent magnitude, $m$ ) of cosmologically-distant indicators whose absolute luminosity (equivalently, the absolute magnitude, $M$ ) is assumed to be known.

SN Ia events constitute one of the most suitable cosmological standard candles. Today, more than 600 SN Ia events have been identified spectroscopically (see, e.g., Suzuki et al. 2012) by a number of scientific groups (see, e.g., Hamuy et al. 1996; Garnavich et al. 1998; Perlmutter et al. 1998, 1999a; Riess et al. 1998, 2001, 2004, 2007; Schmidt et al. 1998; Knop et al. 2003; Tonry et al. 2003; Barris et al. 2004; Krisciunas et al. 2005; Astier et al. 2006; Jha et al. 2006; Miknaitis et al. 2007; WoodVasey et al. 2007; Amanullah et al. 2008, 2010; Holtzman et al. 2008; Kowalski et al. 2008; Hicken et al. 2009a,b; Kessler et al. 2009; Contreras et al. 2010; Guy et al. 2007; Suzuki et al. 2012). In each and every one of these surveys, the SN Ia events appear to be dimmer at peak luminosity, i.e., they seem to lie farther away, than what would have been expected in the context of a dust (i.e., pressureless) Universe.

At this point, we recall that, when spatial flatness was established, the common perception about the cosmos was that the DM constituents are collisionless. Hence the Universe matter content was most favorably interpreted as dust. This inevitably led to the assumption of a dark energy component. However, in a polytropic-DM model, this assumption would have not been necessary, since the appropriate candidate to provide the extra energy needed to flatten the Universe would have already been included in the model (the energy of the internal motions). In this context, we cannot help but wonder whether the observed distribution of the SNe Ia standard candles can be appropriately accommodated in a polytropic-DM model or not.

Many samples of SN Ia data have already appeared in the literature to scrutinize the viability of the various DE scenarios (see, e.g., Davis et al. 2007; Cuhna 2009). In this context, today there is the most extended SN Ia dataset, consisting of 580 events, which is known as the Union 2.1 Compilation (Suzuki et al. 2012). This sample ${ }^{1}$ is an augmented version of the Union 2 SN Ia Compilation (Amanullah et al. 2010) based on the systematic methodology developed by Kowalski et al. (2008). In addition to the Union 2 SN dataset, the Union 2.1 Compilation includes 102 low-redshift SNe Ia from the CfA3 survey (Hicken et al. 2009a), 129 intermediate-redshift SNe Ia (Holtzman et al. 2008), five intermediate-redshift events discovered from La Palma (Amanullah et al. 2008), and six new highredshift SNe Ia data (Suzuki et al. 2012).

To estimate the contribution of the polytropic approach to the DE concept, we shall overplot the corresponding theoreticallyderived distance modulus, $\mu(z)=m-M$, on the Hubble

Available at http://www . supernova.lbl.gov/Union 
( $\mu$ versus $z$ ) diagram of the Union 2.1 SN Compilation. The Kcorrected distance modulus of a light-emitting source is given by

$\mu(z)=5 \log \left(\frac{d_{\mathrm{L}}}{\mathrm{Mpc}}\right)+25$

(see, e.g., Narlikar 1983, Eqs. (13.10) and (13.12), p. 359), where $d_{\mathrm{L}}$ is the luminosity distance of the source, measured in megaparsecs $(\mathrm{Mpc})$. In a spatially-flat model, $d_{\mathrm{L}}$ can be expressed as a function of the cosmological redshift and the Hubble parameter, as follows

$d_{\mathrm{L}}(z)=c(1+z) \int_{0}^{z} \frac{\mathrm{d} z^{\prime}}{H\left(z^{\prime}\right)}$

(see, e.g., Peacock 1999, p. 76). Accordingly, inserting Eq. (68) into Eq. (77), we obtain

$$
\begin{aligned}
d_{\mathrm{L}}(z)= & \frac{c}{H_{0}}(1+z) \\
& \times \int_{0}^{z} \frac{\mathrm{d} z^{\prime}}{\left(1+z^{\prime}\right)^{\frac{3 \Gamma}{2}}\left[\left(1-\Omega_{\mathrm{M}}\right)+\Omega_{\mathrm{M}}\left(1+z^{\prime}\right)^{3(1-\Gamma)}\right]^{1 / 2}} .
\end{aligned}
$$

Once again, the integral on the rhs of Eq. (78) can be solved explicitly in terms of hypergeometric functions (see, e.g., Gradshteyn \& Ryzhik 2007 (7th edn.), pp. 1005-1008), as follows

$$
\begin{aligned}
& d_{L}(z)=\frac{2 c}{H_{0}} \frac{1}{\sqrt{1-\Omega_{\mathrm{M}}}} \frac{1+z}{2-3 \Gamma}\left[(1+z)^{\frac{2-3 \Gamma}{2}}\right. \\
& \quad \times{ }_{2} F_{1}\left(\frac{2-3 \Gamma}{6(1-\Gamma)}, \frac{1}{2} ; \frac{8-9 \Gamma}{6(1-\Gamma)} ;-\left[\frac{\Omega_{\mathrm{M}}}{1-\Omega_{\mathrm{M}}}\right](1+z)^{3(1-\Gamma)}\right) \\
& \left.-{ }_{2} F_{1}\left(\frac{2-3 \Gamma}{6(1-\Gamma)}, \frac{1}{2} ; \frac{8-9 \Gamma}{6(1-\Gamma)} ;-\left[\frac{\Omega_{\mathrm{M}}}{1-\Omega_{\mathrm{M}}}\right]\right)\right]
\end{aligned}
$$

Next, we overplot Eq. (76), with $d_{\mathrm{L}}(z)$ given by Eq. (79) on the $\mu$ versus $z$ diagram of $580 \mathrm{SNe}$ Ia of the extended Union 2.1 Compilation (Suzuki et al. 2012). To do so, we follow Komatsu et al. (2011), admitting that today, $\Omega_{\mathrm{M}}=0.274$ and $H_{0}=$ $70.2 \mathrm{Km} \mathrm{s}^{-1} \mathrm{Mpc}^{1}$; hence, $2 c / H_{0}=8,547 \mathrm{Mpc}$. The outcome is presented in Fig. 5, for several values of the polytropic exponent, $-0.377<\Gamma \leq 0.541$. As expected, the theoretically-derived curves representing the distance modulus, $\mu$, as a function of $z$ in the polytropic-DM model, fit the entire SN Ia distribution much more accurately than the collisionless-DM (EdS) formula (dashed curve), given by

$$
d_{\mathrm{L}}^{\mathrm{EdS}}(z)=\frac{2 c}{H_{0}}(1+z)^{1 / 2}\left[(1+z)^{1 / 2}-1\right]
$$

(see, e.g., Carroll et al. 1992). In this case, from Fig. 6, we see that, the best fit of the theoretically-derived result, given by Eqs. (76) and (79), to the observationally-determined Hubble diagram of the Union 2.1 SN dataset is achieved for $-0.089<$ $\Gamma \leq 0$ (in connection, see next section).

\section{The physics of transition}

\subsection{The velocity of sound}

To determine the velocity of sound in relativistic hydrodynamics, one simply begins with the conservation law, given by

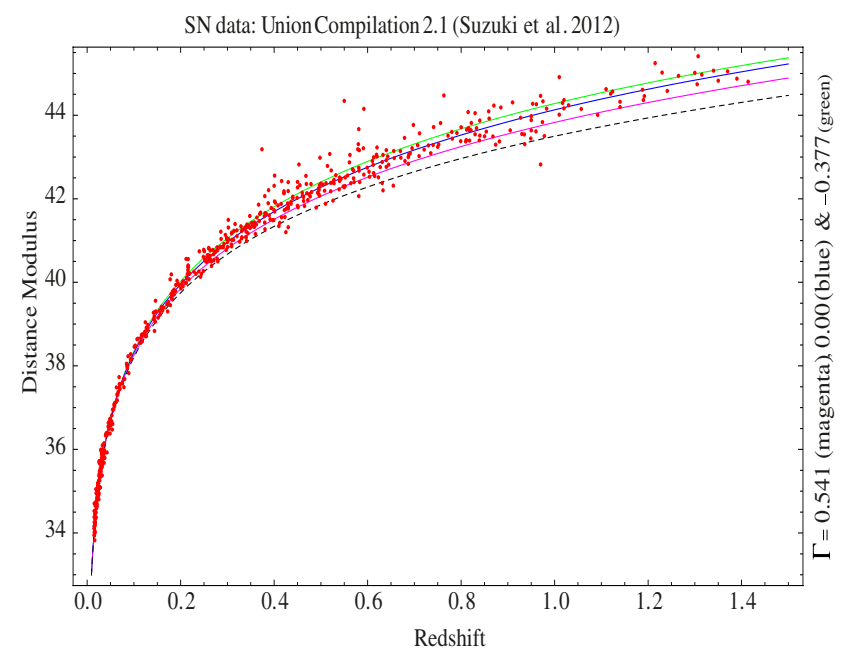

Fig. 5. Hubble diagram of the Union 2.1 SN Compilation (red dots). Overplotted are the theoretical curves, representing the distance modulus as a function of the cosmological redshift in the polytropic-DM model, for $\Omega_{\mathrm{M}}=0.274$ and several (acceptable) values of the polytropic exponent, namely, $\Gamma=0.541$ (magenta), $\Gamma=0$ (blue), and $\Gamma=-0.377$ (green), as compared to the corresponding quantity in the collisionlessDM (dust) case (dashed curve).

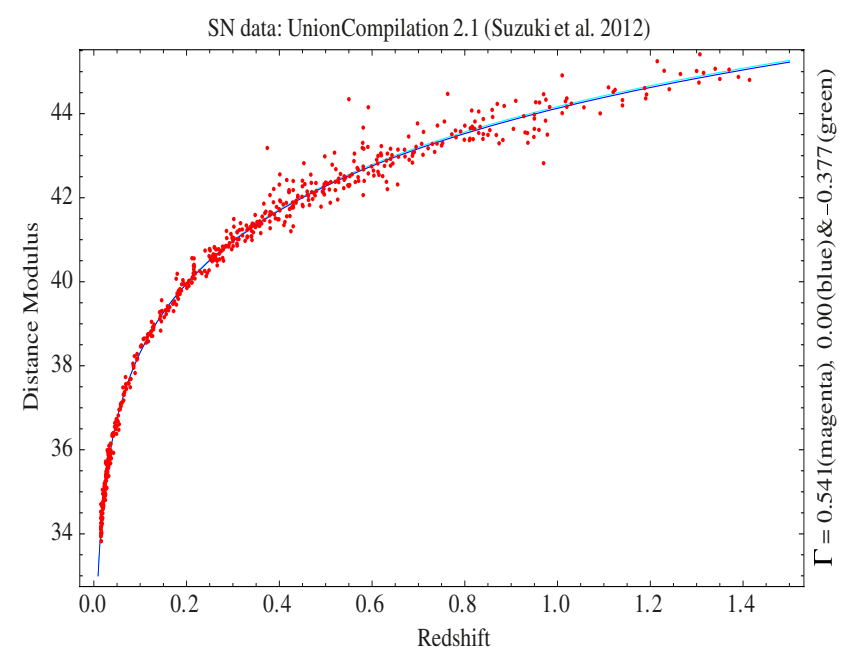

Fig. 6. Observationally-determined Hubble diagram of the Union 2.1. Compilation. Overplotted are the best-fit curves, which are too close to be distinguished, representing the function $\mu(z)$ in the polytropic-DM model, when $-0.089<\Gamma \leq 0$.

Eq. (24), and monitors its response to infinitesimal compressions or expansions of the fluid. To linear perturbation terms, the resulting wave equation

$\frac{\partial^{2} \delta}{\partial t^{2}}-c^{2}\left(\frac{\partial p}{\partial \varepsilon}\right)_{\mathcal{S}} \nabla^{2} \delta=0$,

where $\delta=\frac{\delta n}{n}$ is the particles' number density contrast, defines the isentropic velocity of sound as

$c_{\mathrm{s}}^{2}=c^{2}\left(\frac{\partial p}{\partial \varepsilon}\right)_{\mathcal{S}}$

(see, e.g., Weinberg 1972, p. 52). In view of Eq. (82), barotropic flow in a polytropic-DM perfect fluid, defined by Eqs. (3), (33) and (39), suggests that in the cosmological model under consideration, the velocity of sound may no longer be constant, 
but a function of the cosmological redshift, parametrized by $\Gamma$. Accordingly, we distinguish two cases:

(i) $\Gamma=0$ : in this case, $p=p_{0}=$ const., and, therefore,

$c_{\mathrm{S}}^{2}(\Gamma=0)=0$.

In other words, in an isobaric cosmological model, no acoustic waves ever propagate; the Universe remains "silent".

(ii) $\Gamma \neq 0$ : in this case, by virtue of Eqs. (3), (33) and (39), the total energy density of the Universe matter-energy content is written in the form

$$
\varepsilon=\underbrace{\rho c^{2}}_{\varepsilon_{\mathrm{mat}}}+\underbrace{\frac{p}{\Gamma-1}}_{\varepsilon_{\mathrm{int}}}=\rho_{0} c^{2}\left(\frac{p}{p_{0}}\right)^{1 / \Gamma}+\frac{p}{\Gamma-1} .
$$

Partial differentiation of Eq. (84) with respect to $\varepsilon$, yields

$\left(\frac{\partial p}{\partial \varepsilon}\right)_{\mathcal{S}}=\frac{\Gamma\left(\frac{p}{\rho c^{2}}\right)}{1+\frac{\Gamma}{\Gamma-1}\left(\frac{p}{\rho c^{2}}\right)}=\left(\frac{c_{\mathrm{s}}}{c}\right)^{2}$.

Accordingly, the velocity of sound as a function of the cosmological redshift, is given by

$\left(\frac{c_{\mathrm{S}}}{c}\right)^{2}=-\frac{\Gamma(1-\Gamma) \frac{1-\Omega_{\mathrm{M}}}{\Omega_{\mathrm{M}}}}{(1+z)^{3(1-\Gamma)}+\Gamma \frac{1-\Omega_{\mathrm{M}}}{\Omega_{\mathrm{M}}}}$.

In view of Eq. (55), the denominator on the rhs of Eq. (86) is positive for every $z \geq 0$, and, therefore, the condition of a positive velocity-of-sound square yields a major constraint on the upper bound of $\Gamma$, namely,

$\left(\frac{c_{\mathrm{s}}}{c}\right)^{2}>0 \Leftrightarrow \Gamma<0$.

Hence, in what follows, we consider $\pm \Gamma=\mp|\Gamma|$. Now, Eq. (86) is written in the form

$\left(\frac{c_{\mathrm{S}}}{c}\right)^{2}=(1+|\Gamma|) \frac{|\Gamma| \frac{1-\Omega_{\mathrm{M}}}{\Omega_{\mathrm{M}}}}{(1+z)^{3(1+|\Gamma|)}-|\Gamma| \frac{1-\Omega_{\mathrm{M}}}{\Omega_{\mathrm{M}}}}$.

With respect to $z$, there are two values of $\left(\frac{c_{\mathrm{s}}}{c}\right)^{2}$ of particular interest, namely, (a) at transition $\left(z=z_{\text {tr }}\right)$, where

$\left(\frac{c_{\mathrm{s}}}{c}\right)_{\mathrm{tr}}^{2}=\frac{|\Gamma|}{2} \Rightarrow|\Gamma|=2\left(\frac{c_{\mathrm{s}}}{c}\right)_{\mathrm{tr}}^{2}$,

attributing to the polytropic exponent an unexpected physical interpetation, and $(\mathrm{b})$ at the present epoch $(z=0)$, when $\left(\frac{c_{\mathrm{s}}}{c}\right)^{2}$ attains its maximum value, namely,

$\left(\frac{c_{\mathrm{s}}}{c}\right)_{0}^{2}=(1+|\Gamma|) \frac{|\Gamma| \frac{1-\Omega_{\mathrm{M}}}{\Omega_{\mathrm{M}}}}{1-|\Gamma| \frac{1-\Omega_{\mathrm{M}}}{\Omega_{\mathrm{M}}}}$.

At this point, we recall that, for relativistic particles (i.e., HDM), the velocity of sound reads $\left(\frac{c_{\mathrm{s}}}{c}\right)^{2}=\frac{1}{3}$ (see, e.g., Weinberg 1972, p. 51; Landau \& Lifshitz 1987, p. 509). Accordingly, the cosmological requirement for CDM at the present epoch, is translated to

$\left(\frac{c_{\mathrm{s}}}{c}\right)_{0}^{2}<\frac{1}{3}$ which, in the polytropic-DM model under consideration, results in

$|\Gamma|^{2} \frac{1-\Omega_{\mathrm{M}}}{\Omega_{\mathrm{M}}}+\frac{4}{3}|\Gamma| \frac{1-\Omega_{\mathrm{M}}}{\Omega_{\mathrm{M}}}-\frac{1}{3}<0$,

yielding

$|\Gamma|<\frac{2}{3}\left[\sqrt{1+\frac{3}{4} \frac{\Omega_{\mathrm{M}}}{1-\Omega_{\mathrm{M}}}}-1\right] \overbrace{=}^{\Omega_{\mathrm{M}}=0.274} 0.089$.

The physical requirements given by Eqs. (87) and (91), together with Eq. (83), have led to an even narrower range of values of the only free parameter of this model, namely,

$-0.089<\Gamma \leq 0$,

i.e., in a realistic polytropic-DM cosmological model, the polytropic exponent, if not zero (i.e., a $\Lambda \mathrm{CDM}$-equivalent model), is definitely negative and very close to zero (in connection, see Fig. 6). The result given by Eq. (94) lies well within the corresponding range obtained for a generalized Chaplygin gas ( $p \sim-\rho^{-\alpha}$ ), from the analysis of X-ray and SN Ia measurements, in connection to data from Fanaroff-Riley type IIb radiogalaxies, namely, $\alpha=-0.09_{-0.33}^{+0.54}$ (Zhu 2004).

In view of Eq. (94), Eq. (89) yields

$0 \leq\left(\frac{c_{\mathrm{s}}}{c}\right)_{\operatorname{tr}}^{2}<0.044$

while, by virtue of Eq. (71), the present-time value of the deceleration parameter is maintained at

$-0.686<q_{0} \leq-0.589$.

Equation (96) has a well-shaped cross section with the lower part of the observationally-determined range of values of $q_{0}$ (based on the SALT2 fitting to the $\mathrm{SNe}+\mathrm{BAO} / \mathrm{CMB}$ data), namely, $q_{0}=$ $-0.53_{-0.13}^{+0.17}$ (Giostri et al. 2012).

Furthermore, the combination of Eqs. (6) and (94) results in a most definite set of allowed values of the heat capacity, $C$ (in units of $C_{\mathrm{V}}$ ), in a polytropic (DM) Universe, namely, those in the range

$0.082+0.918 \gamma<\frac{C}{C_{\mathrm{V}}} \leq \gamma$,

which, for $1<\gamma \leq 3$ (see, e.g., Guidry 1998, p. 131), is depicted in Fig. 7 (blue-filling region).

\subsection{Observables at transition}

As we saw in Sect. 2 , for $\Gamma<1$, the work done by the pressure along a polytropic process in an expanding Universe, is given back to the cosmic fluid itself. As a consequence, both the internal energy, $\mathcal{U}$, and the temperature, $T$, of the polytropic-DM fluid increase toward the present epoch. It is straightforward to prove that the change of $\mathcal{U}$ and $T$ from some $z$ to $z=0$ is given by

$$
\begin{aligned}
\left.\frac{\Delta \mathcal{U}}{\mathcal{U}}\right|_{z \rightarrow 0} & =\frac{\mathcal{U}_{0}-\mathcal{U}(z)}{\mathcal{U}_{0}}=1-\frac{1}{(1+z)^{3(1+|\Gamma|)}} \\
& =\frac{T_{0}-T(z)}{T_{0}}=\left.\frac{\Delta T}{T}\right|_{z \rightarrow 0},
\end{aligned}
$$

which, at $z=z_{\text {tr }}$, results in

$\frac{T_{\mathrm{tr}}}{T_{0}}=\left(\frac{\Omega_{\mathrm{M}}}{1-\Omega_{\mathrm{M}}}\right) \frac{1}{2+3|\Gamma|}=\frac{0.377}{2+3|\Gamma|}$. 


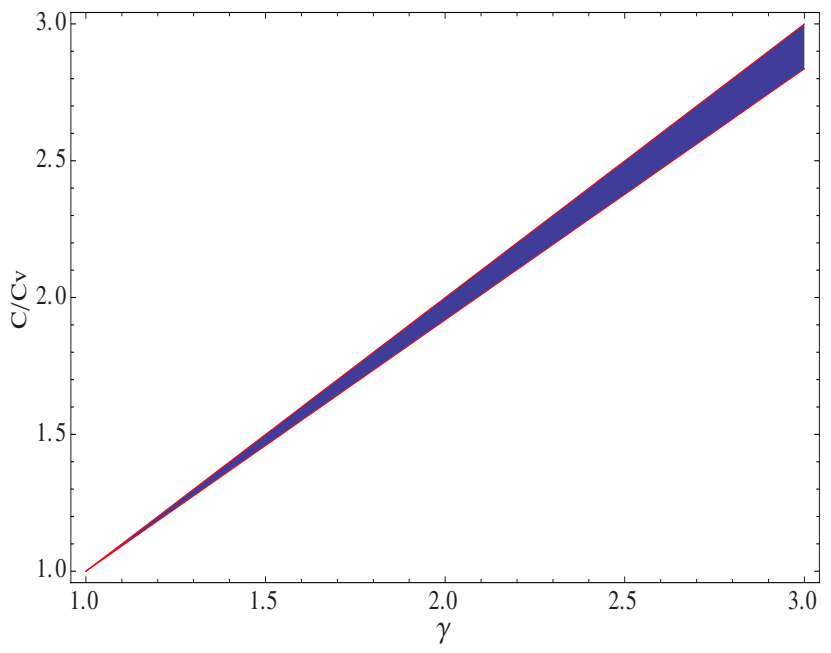

Fig. 7. Allowed set of values of the heat capacity $C$ (in units of $C_{\mathrm{V}}$ ) in a polytropic-DM model with $-0.089<\Gamma \leq 0$ (blue-filling region), as a function of the adiabatic index $1<\gamma \leq 3$.

From Eq. (99), we find that, for $-0.089<\Gamma \leq 0$, the temperature of DM at transition, in units of its present-time value, lies in the range

$0.165<\frac{T_{\text {tr }}}{T_{0}} \leq 0.189$

a prediction to be verified by observation. Of course, the exact value of $T_{0}$ depends on the exact nature of the DM constituents. Notice, however, that, $T_{0}$ cannot exceed the presenttime cosmic neutrino background temperature, $T_{v}=1.95 \mathrm{~K}$, in order for the Big Bang nucleosynthesis to remain unaffected (see, e.g., Lundgren et al. 2010). Accordingly, admitting $T_{0}=$ $1.95 \mathrm{~K}$ as the upper bound of the DM temperature at the present epoch, we find that the corresponding lower bound at transition is $0.322 \mathrm{~K}<T_{\text {tr }} \leq 0.369 \mathrm{~K}$.

By virtue of Eq. (100), we can use Eq. (10), to determine the variation of entropy, along a polytropic change of the DM fluid, from the epoch of transition to the present epoch. We obtain

$1.666 C \leq\left.\Delta \mathcal{S}\right|_{z_{\mathrm{tr}} \rightarrow 0}=\mathcal{S}_{0}-\mathcal{S}_{\mathrm{tr}}<1.802 C$,

where $C$ is given by Eq. (97).

Finally, in view of Eq. (87), the combination of Eqs. (52) and (72) yields

$\frac{\varepsilon_{\mathrm{int}}}{\varepsilon_{\mathrm{mat}}}=\frac{1}{2+3|\Gamma|}\left(\frac{1+z_{\mathrm{tr}}}{1+z}\right)^{3(1+|\Gamma|)}$,

which, at $z=z_{\text {tr }}$, results in

$\left.\frac{\varepsilon_{\text {int }}}{\varepsilon_{\text {mat }}}\right|_{\text {tr }}=\frac{1}{2+3|\Gamma|}$

In contrast to the common perception, in a polytropic-DM model the onset of transition from deceleration to acceleration does not necessarily require $\varepsilon_{\text {int }}>\varepsilon_{\text {mat }}$. In fact, according to Eq. (52), equality between the internal (dark) energy density and its restmass counterpart occurred quite later, at $z=0.384$ (for $\Gamma=0$ ), which is in good agreement with the corresponding observational $(\Lambda \mathrm{CDM})$ result, namely, $z=0.391 \pm 0.033$ (Suzuki et al. 2012). Depending on $\Gamma$ (in the range given by Eq. (94)),
Eq. (103) suggests that, the transition from deceleration to acceleration takes place when

$0.441<\frac{\varepsilon_{\text {int }}}{\varepsilon_{\text {mat }}} \leq 0.500$.

The question is why it happens this way. The answer is both revealing and simple: because of the GR itself!

\subsection{Why and when the Universe transits to acceleration}

In the context of GR, the dynamics of a homogeneous and isotropic, spatially-flat cosmological model, such as the one given by Eq. (22), is completely determined by Eqs. (30) and (40). Combining these two equations together, we obtain

$\frac{\ddot{S}}{S}=-\frac{4 \pi G}{3 c^{2}}(\epsilon+3 p)$

(see, e.g., Linder 2008; Caldwell \& Kamionkowski 2009) and, hence, the condition for accelerated expansion, $\ddot{S}>0$, is, in fact, translated to

$\epsilon+3 p<0$.

Inserting Eqs. (3) and (39) of our (polytropic-DM) model into Eq. (106), and if we also take Eqs. (33), (45), (51) and (87) into account, we find that the condition for the acceleration of the cosmological model (22) results in

$$
\begin{aligned}
\varepsilon+3 p= & \rho_{0} c^{2}(1+z)^{3} \\
& \times\left[1-(2+3|\Gamma|) \frac{1-\Omega_{\mathrm{M}}}{\Omega_{\mathrm{M}}} \frac{1}{(1+z)^{3(1+|\Gamma|)}}\right]<0 .
\end{aligned}
$$

Hence, a homogeneous and isotropic, spatially-flat cosmological model filled with matter in the form of a polytropic (DM) perfect fluid, most definitely accelerates its expansion, at cosmological redshifts lower than a specific value given by

$z<\left[(2+3|\Gamma|) \frac{1-\Omega_{\mathrm{M}}}{\Omega_{\mathrm{M}}}\right]^{\frac{1}{3(1+\Gamma \Gamma)}}-1 \equiv z_{\mathrm{tr}}$,

in complete agreement to the transition redshift, $z_{\text {tr }}$, defined (in an independent manner) via Eq. (72).

So, we conclude that to determine the "why" and "when" of the onset of cosmic acceleration, there may be no need for any exotic DE at all. Equivalently, a polytropic cosmic fluid could most definitely reveal such a reality, and, at the same time, it would illuminate the nature of the long sought DE, as due to the cosmic fluid's internal motions, whose catalytic role should be particularly emphasized.

\subsection{Additional constraints from $C M B$}

To tighten up the constraints on the various DE models, a common approach is to include additional information from the $\mathrm{CMB}$, in the form of the so-called shift parameter, $R$, which is related to the position of the first acoustic peak in the power spectrum of the temperature anisotropies (Efstathiou \& Bond 1999). This parameter is not a directly-measured quantity, however. It is derived from the observational data only after assuming a specific model, usually the spatially-flat $\Lambda \mathrm{CDM}$ model. Therefore, extra care is needed when using $R$ to test more exotic DE models (see, e.g., Elgarøy \& Multamäki 2007). 
The use of the shift parameter as a probe of DE is based on the observation that different models will result in almost identical CMB power spectra (Efstathiou \& Bond 1999), if they have: (i) Identical CDM densities $\omega_{\mathrm{c}}=\Omega_{\mathrm{c}} h^{2}$ ( $h$ is the dimensionless Hubble constant, defined by $H_{0}=100 \mathrm{~h} \mathrm{~km} \mathrm{~s}^{-1} \mathrm{Mpc}^{-1}$ ); (ii) identical baryonic densities $\omega_{\mathrm{b}}=\Omega_{\mathrm{b}} h^{2}$; (iii) identical primordial fluctuation spectra; and (iv) identical values of the shift parameter, which, as far as spatially-flat models are concerned, is given by

$R=\sqrt{\Omega_{\mathrm{M}}} \int_{0}^{z_{*}} \frac{\mathrm{d} z}{H(z) / H_{0}}$,

where $z_{*}$ the value of the cosmological redshift at photon decoupling.

In view of Eqs. (68) and (94), in the polytropic-DM cosmological model under consideration Eq. (109) is written in the form

$R=\int_{0}^{z_{*}} \frac{\left(1+z^{\prime}\right)^{\frac{3}{2}|\Gamma|} \mathrm{d} z^{\prime}}{\left[\left(1-\Omega_{\mathrm{M}}\right)+\Omega_{\mathrm{M}}\left(1+z^{\prime}\right)^{3(1+|\Gamma|)}\right]^{1 / 2}}$

Once again, the integral on the rhs of Eq. (110) can be solved explicitly in terms of hypergeometric functions (see e.g., Gradshteyn \& Ryzhik 2007 (7th edn.), pp. 1005-1008), as follows

$$
\begin{aligned}
R= & \frac{2}{(2+3|\Gamma|) \sqrt{1-\Omega_{\mathrm{M}}}}\left[\left(1+z_{*}\right)^{\frac{2+3|\Gamma|}{2}}\right. \\
& \times{ }_{2} F_{1}\left(\frac{2+3|\Gamma|}{6(1+|\Gamma|)}, \frac{1}{2} ; \frac{8+9|\Gamma|}{6(1+|\Gamma|)} ;\right. \\
& \left.-\left[\frac{\Omega_{\mathrm{M}}}{1-\Omega_{\mathrm{M}}}\right]\left(1+z_{*}\right)^{3(1+|\Gamma|)}\right) \\
& \left.-{ }_{2} F_{1}\left(\frac{2+3|\Gamma|}{6(1+|\Gamma|)}, \frac{1}{2} ; \frac{8+9|\Gamma|}{6(1+|\Gamma|)} ;-\left[\frac{\Omega_{\mathrm{M}}}{1-\Omega_{\mathrm{M}}}\right]\right)\right] .
\end{aligned}
$$

As far as the value of $z_{*}$ is concerned, we adopt the nine-year WMAP survey final result (Bennett et al. 2013), $z_{*}=1091.64 \pm$ 0.47 , at $68 \%$ confidence level (CL). Accordingly, for $\Gamma=0$, i.e., in the $\Lambda$ CDM-equivalent polytropic case, Eq. (111) yields

$R=1.7342$,

while, according to the nine-year WMAP survey final results (Bennett et al. 2013), the value of the shift parameter in the standard $\Lambda$ CDM model is

$$
R=1.7329 \pm 0.0058(68 \% \mathrm{CL}) \text {. }
$$

In other words, the value of the shift parameter in the $\Lambda \mathrm{CDM}$ limit of our (polytropic-DM) model reproduces, to high accuracy, the corresponding result obtained by fitting the observational (CMB) data to the standard $\Lambda \mathrm{CDM}$ model.

Although applicable only to $\Lambda$ CDM-like models, the nineyear WMAP survey final result given by Eq. (113) is compatible also with our $\Gamma \neq 0$ model, up to $|\Gamma| \leq 0.0271$ (Fig. 8). On the other hand, the range of values of $R$, arising from the combination of the Planck first-data release with those of the WMAP survey (Wang \& Wang 2013), namely,

$R=1.7407 \pm 0.0091(68 \% \mathrm{CL})$,

encapsulates the whole range of allowed values of $\Gamma$ given by Eq. (94), yielding as the most preferable the value $|\Gamma|=0.040$

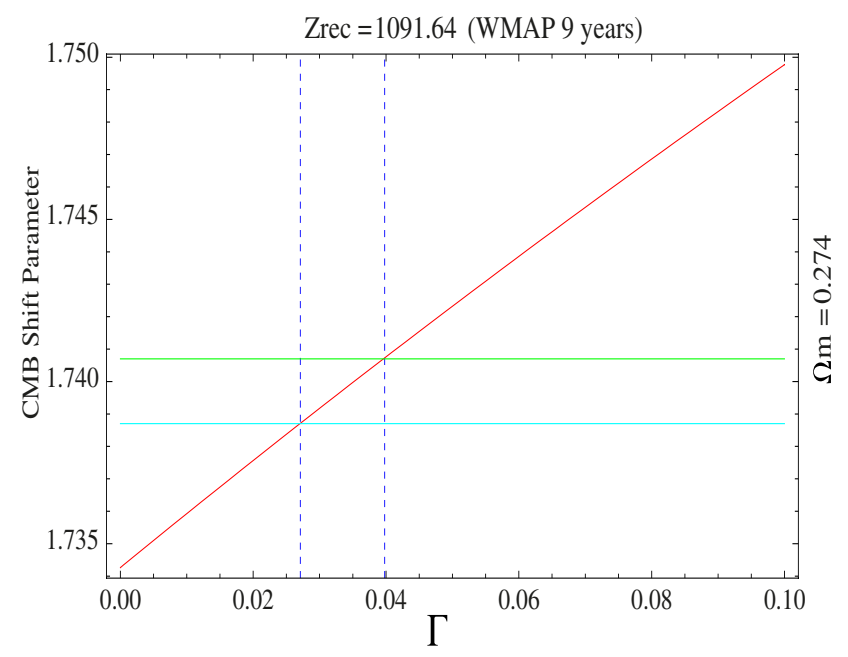

Fig. 8. Theoretically (in the context of a polytropic-DM model) determined CMB-shift parameter, Eq. (111), as a function of the polytropic exponent $-0.089<\Gamma \leq 0$ (red solid line). The cyan horizontal straight line denotes the upper bound (at $68 \% \mathrm{CL}$ ) of the $R$-value, obtained from the nine-year WMAP survey final results, namely, $R=1.7387$, and the corresponding green line denotes the mean value of $R$, arising from the combination of the Planck first-data release with those of the WMAP survey, namely, $R=1.7407$. The vertical dashed lines denote the upper bound of $\Gamma(|\Gamma|=0.0271)$, arising from WMAP alone, and the corresponding most preferable value $(|\Gamma|=0.0398)$, arising from the combination of the Planck first-data release with those of the WMAP survey.

(Fig. 8). This result conforms with the final conclusion of Wang \& Wang (2013) that the addition of Planck distance priors to the current cosmological data leads to a marginal inconsistency with a cosmological constant in a spatially-flat Universe.

In view of the aforementioned results, to use the shift parameter as a constraint on a DE model, then, first of all, we should derive the corresponding distribution from the CMB data. This cannot be done without preassuming a model, i.e., without predetermining the primordial power spectrum of density fluctuations since they form the basis for calculating the $\mathrm{CMB}$ anisotropies, and, therefore, making implicit assumptions about inflation. Even so, the value of the CMB-shift parameter in the $\Lambda$ CDM limit of our (polytropic-DM) model (i.e., for $\Gamma=0$ ) reproduces to high accuracy the corresponding result obtained by fitting the observational $(\mathrm{CMB})$ data to the standard $\Lambda \mathrm{CDM}$ model.

\section{Discussion}

We have examined the possibility that the dark energy needed to flatten the Universe is represented by the energy of the internal motions of a polytropic perfect fluid. Polytropic processes in a DM fluid have been most successfully used in modeling dark galactic haloes, significantly improving the velocity dispersion profiles of galaxies. Motivated by these results, we have explored the physical and dynamical characteristics, as well as the evolution of a cosmological model driven by a gravitating fluid with thermodynamical content, consisting of DM (dominant) and baryonic matter (subdominant).

In the distant past, the matter-energy content of this model behaves as a pressureless fluid (cf. Eq. (70), for $z \gg 1$ ). However, toward the present epoch, the internal physical characteristics of this fluid (i.e., beyond its rest-mass density) take over (cf. Eq. (20)), yielding the DM itself thermodynamically 
involved. In fact, at cosmological redshifts lower than $z=0.384$, $\varepsilon_{\text {int }}>\varepsilon_{\text {mat }}$, i.e., the energy density of the internal motions dominates over the corresponding rest-mass quantity.

In this context, the fundamental matter constituents of this model are the volume elements of the DM fluid performing polytropic flows. As a consequence, we also took the energy of this fluid's internal motions into account as a source of the universal gravitational field. In this way, we have been able to determine the appropriate form of the cosmic scale factor (see Fig. 3), which (under the assumption that the DM is thermodynamically involved) governs the evolution of the Universe, being modeled as a spatially-flat RW spacetime. Accordingly, we have asked ourselves whether this model can accommodate both the observed distribution of the cosmologically-distant indicators and the associated phase of accelerated expansion.

Our findings are, in fact, quite promising. In principle, the energy of the internal motions of the polytropic-DM fluid can account for the extra DE, so that, at the present epoch, the total energy density parameter, $\Omega=\frac{\varepsilon}{\varepsilon_{c}}$, equals to unity (cf. Eq. (50)). Furthermore, for values of the polytropic exponent, $\Gamma$, lower than 0.541, the pressure of the DM fluid becomes negative enough (cf. Eq. (45)), in the sense that, the Universe accelerates its expansion at cosmological redshifts smaller than a transition value (cf. Eq. (72)), in a way also consistent with the requirement $\varepsilon+3 p<0$ (cf. Eqs. (107) and (108)).

It is both pedagogic and quite interesting to stress the various reasons for imposing successive constraints on the exact value of the polytropic exponent, $\Gamma$. More specifically, the second law of thermodynamics in an expanding Universe suggests that $\Gamma \leq \gamma$ (cf. Eq. (13)). In this context, for the pressure work to be negative, i.e., to be attributed to the cosmic fluid itself, $\Gamma<1$ (cf. Eq. (20)). Furthermore, the condition $z_{\text {tr }} \geq 0$ suggests that $\Gamma \leq 0.541$ (cf. Eq. (74)), while, the condition $p_{0}>-\varepsilon_{0}$ (i.e., the requirement of a non-phantom Universe) implies that $\Gamma>-0.377$ (cf. Eq. (55)). Moreover, the positivity of the velocity-of-sound square at all $z$ imposes the major constraint $\Gamma \leq 0$ (cf. Eqs. (83) and (87)), and, finally, the requirement for CDM at the present epoch implies that $\Gamma>-0.089$ (cf. Eq. (93)).

In view of the aforementioned results, a realistic polytropic (DM) cosmological model (i.e., one that is compatible with the fundamental physical laws and the basic mathematical principles, and, at the same time, is compatible with the observational data currently available) requires that, eventually, the polytropic exponent, $\Gamma$, should be settled down to the range $-0.089<\Gamma \leq 0$, namely, if it is not zero, it is definitely negative and very close to zero (in connection, see Fig. 6). In any case, the polytropic-DM model attributes a well-posed physical meaning to $\Gamma$ itself, suggesting that its absolute value is, in fact, a representation of the value of $\left(\frac{c_{\mathrm{s}}}{c}\right)^{2}$ at transition (cf. Eq. (89)).

In the context of the polytropic DM model, the ( $\Gamma$-dependent) present-time value of the deceleration parameter (Eq. (96)), is in good agreement with the observationallydetermined range of values of $q_{0}$, namely, $q_{0}=-0.53_{-0.13}^{+0.17}$ (Giostri et al. 2012). For $\Gamma=0$, the equality between the internal (dark) energy density and its rest-mass counterpart occurs at $z=0.384$ (cf. Eq. (52)), a theoretical prediction that quite accurately reproduces the corresponding observational $(\Lambda \mathrm{CDM})$ result, namely, $z=0.391 \pm 0.033$ (Suzuki et al. 2012).

Moreover, our cosmological model does not suffer either from the age problem (see Fig. 2) or from the coincidence problem (cf. Eqs. (52) and (53)), and, at the same time, it reproduces to high accuracy the distance measurements, performed with the aid of the SNe Ia standard candles (see Fig. 6). Furthermore, the value of the CMB-shift parameter in the $\Lambda$ CDM limit of our (polytropic-DM) model (i.e., for $\Gamma=0$ ) reproduces, to high accuracy the corresponding value obtained by fitting the observational (CMB) data to the standard $\triangle \mathrm{CDM}$ model (cf. Eqs. (112) and (113)). Finally, our polytropic-DM model most naturally interprets not only when, but also why the Universe transits from deceleration to acceleration (cf. Eqs. (107) and (108)), and reveals the catalytic role of the internal motions in such a transition, i.e., for the onset of acceleration, the internal-energy density suffices to be half the value of the corresponding rest-mass quantity (cf. Eq. (104)).

Nevertheless, there are also several aspects of the polytropicDM model, that still remain open, such as:

- The cosmological behavior of the corresponding density perturbations: since we do not neglect the pressure with respect to the energy density, the perturbations should be studied in a general-relativistic way. In this case, a problem arises on the choice of gauge. As it has already been recognized by Gondolo \& Freese (2003), in a polytropic model the value of the density perturbation depends on the choice of gauge, even well-into the matter-dominated era. This creates a problem of interpretation for fluctuations in the present Universe, which must be addressed in future studies.

- The origin of the (extra) amount of heat, $C d T$, offered to the thermodynamical system (cf. Eq. (21)): Skillfully, this could be attributed to a long-range confining force between the DM constituents (see, e.g., Gondolo \& Freese 2003; ArkaniHamed et al. 2009; Van den Aarssen et al. 2012), which, in our case, would be of the form $F=-K r^{2+3|\Gamma|}$, where $r$ is the radial distance and $K>0$ is a normalization constant (in connection, see Eq. (80) and the discussion following Eq. (89) of Gondolo \& Freese 2003). This force may be of gravitational origin or it may be a new force. However, it is not clear at all that a system subject to a long-range confining force can reach thermodynamic equilibrium, hence, this matter must also be addressed in future studies.

In any case, the assumption that the Universe matter content can be collisional, in the sense that its DM component possesses some sort of thermodynamical content, is to be seen as a natural effort to take into account all the internal physical characteristics of a classical cosmological fluid as sources of the universal gravitational field. Although speculative, the idea that the DE could be attributed to the internal motions of a polytropic-DM fluid is intriguing and should be further explored and scrutinized.

Acknowledgements. The authors would like to thank Dr. Spyros Basilakos, for illuminating discussions and his useful comments on the content of this article. Financial support by the Research Committee of the Technological Education Institute of Central Macedonia at Serres, Greece, under grant SAT/ME/230113$10 / 04$, is gratefully acknowledged.

\section{References}

Abramowitz, M., \& Stegun, I. 1970, Handbook of mathematical functions (New York: Dover)

Adriani, O., Barbarino, G. C., Bazilevskaya, G. A., et al. 2009, Nature, 458, 607 Allen, S. W., Schmidt, R. W., Ebeling, H., Fabian, A. C., \& van Speybroeck, L. 2004, MNRAS, 353, 457

Amanullah, R., Stanishev, V., Goobar, A., et al. 2008, A\&A, 486, 375

Amanullah, R., Lidman, C., Rubin, D., et al. (Supernova Cosmology Project Group) 2010, ApJ, 716, 712

Arkani-Hamed, N., Finkbeiner, D. P., Slatyer, T. R., \& Weiner, N. 2009, Phys. Rev. D, 79, 015014

Armendariz-Picon, C., Mukhanov, V. F., \& Steinhardt, P. J. 2001, Phys. Rev. D, 63, 103510

Astier, P., Guy, J., Regnault, N., et al. 2006, A\&A, 447, 31 
Asadzadeh, S., Safari, Z., Karami, K., \& Abdolmaleki, A. 2014, Int. J. Theor Phys., 53, 1248

Balakin, A. B., Pavón, D., Schwarz, D. J., \& Zimdahl, W. 2003, New J. Phys., 5, 85

Barger, V., Keung, W. Y., Marfatia, D., \& Shaughnessy, G. 2008, Phys. Lett. B, 672,141

Barris, B., Tonry, J. L., Blondin, S., et al. 2004, ApJ, 602, 571

Basilakos, S., \& Plionis, M. 2009, A\&A, 507, 47

Basilakos, S., \& Plionis, M. 2010, Interactive Dark Matter as an alternative to Dark Energy, in Invisible Universe, AIP Conf. Proc., 1241, 721

Bharadwaj, S., \& Kar, S. 2003, Phys. Rev. D, 68, 023516

Baushev, A. N. 2009, MNRAS, 398, 783

Bennett, C. L., Larson, D., Weiland, J. L., et al. 2013, ApJS, 208, 20

Bergstrom, L., Bringmann, T., \& Edsjo, J. 2008, Phys. Rev. D, 78, 103520

Bean, R., \& Doré, O. 2003, Phys. Rev. D, 68, 023515

Bento, M. C., Bertolami, O., \& Sen, A. A. 2002, Phys. Rev. D, 66, 043507

Bilić, N., Tupper, G. B., \& Viollier, R. D. 2002, Phys. Lett. B, 535, 17

Böhmer, C. G., \& Harko, T. 2007, JCAP, 6, 25

Bond, J. R., \& Szalay, A. S. 1983, ApJ, 274, 443

Bond, J. R., Efstathiou G., \& Silk, J. 1980, Phys. Lett., 45, 1980

Boughn, S., \& Crittenden, R. 2004, Nature, 427, 45

Bousso, R., \& Polchinski, J. 2000, JHEP 0006,

Caldwell, R. R. 2002, Phys. Lett. B, 545, 23

Caldwell, R. R., \& Kamionkowski, M. 2009, Annual Rev. Nucl. Part. Sci., 59, 397

Caldwell, R. R., Dave, R., \& Steinhardt, P. J. 1998, Phys. Rev. Lett., 80, 1528

Capozziello, S., Carloni, S., \& Troisi, A, 2003, Recent Res. Dev. Astron. Astrophys., 1, 625

Carroll, S. M., Press, W. H., \& Turner, M. S. 1992, ARA\&A, 30, 499

Chandrasekhar, S. 1939, An Introduction to the Study of Stellar Structure (Chicago University Press)

Chavanis, P-H. 2014, Eur. Phys. J. Plus, 129, 38

Chavanis, P-H. 2012a [arXiv: 1208.0801]

Chavanis, P-H. 2012b [arXiv: 1208.1192]

Cholis, I., Goodenough, L., Hooper, D., Simet, M., \& Weiner, N. 2009a, Phys. Rev. D, 80, 123511

Cholis, I., Dobler, G., Finkbeiner, D. P., Goodenough, L., \& Weiner, N. 2009b, Phys. Rev. D, 80, 123518

Christensen-Dalsgard, J. 2008, Lecture notes on stellar structure and evolution, 6th edn. (Aarhus University Press)

Christians, J. 2012, Int. J. Mech. Eng. Edu., 40, 1 (Manchester Univ. Press)

Cirelli, M., \& Strumia, A. 2008 [arXiv: 0808. 3867]

Cirelli, M., Kadastik, M., Raidal, M., \& Strumia, A. 2009, Nucl. Phys. B, 813, 1

Cohen, T., \& Zurek, K. 2010, Phys. Rev. Lett., 104, 101301

Cohen, A. G., Kaplan, D. M., \& Nelson, A. G. 1999, Phys. Rev. Lett. 82, 4971

Contreras, C., Hamuy, M., Phillips, M. M., et al. 2010, AJ, 139, 519

Copeland, E. J., Sami, M., \& Tsujikawa, S. 2006, Int. J. Mod. Phys. D, 15, 1753

Croft, R. A. C., Weinberg, D. H., Pettini, M., Hernquist, L., \& Katz, N. 1999, ApJ, 520, 1

Cunha, J. V. 2009, Phys. Rev. D, 79, 047301

Davis, T. M., Mörtsell, E., Sollerman, J., et al. 2007, ApJ, 666, 716

de Bernardis, P., Ade, P. A. R., Bock, J. J., et al. (BOOMERanG Collaboration) 2000, Nature, 404, 955

Deffayet, C., Dvali, G., \& Gabadadze, G. 2002, Phys. Rev. D, 65, 044023

Dunkley, J., Komatsu, E., Nolta, M. R., et al. 2009, ApJS, 180, 306

Dutta, S., \& Scherrer, R. J. 2010, Phys. Rev. D, 82, 043526

Dvali, G. R., Gabadadze, G., \& Porratti, M. 2000, Phys. Lett. B, 485, 208

Eisenstein, D. J., Zehavi, I., Hogg, D. W., et al. 2005, ApJ, 633, 560

Efstathiou, G., \& Bond, J. R. 1999, MNRAS, 30, 75

Elgarøy, Ø., \& Mutamäki, T. 2007, A\&A, 471, 65

Esposito-Farese, G., \& Polarski, D. 2001, Phys. Rev. D, 63, 063504

Fardon, R., Nelson, A. E., \& Weiner, N. 2004, JCAP, 10, 005

Feng, J. L., Kaplinghat, M., \& Yu H-B. 2010, Phys. Rev. Lett., 104, 151301

Fock, V. 1959, The theory of space, time and gravitation (London: Pergamon Press)

Fornasa, M., Pieri, L., Bertone, G., \& Branchini, E. 2009, Phys. Rev. D, 80, 023518

Fox, P. J., \& Poppitz, E. 2009, Phys. Rev. D, 79, 083528

Freese, K. 2005, New Astron. Rev., 49, 103

Freese, K., \& Lewis, M. 2002, Phys. Lett. B, 540, 1

Frieman, J. A., Turner, M. S., \& Huterer, D. 2008, ARA\&A, 46, 385

Garnavich, P. M., Jha, S., Challis, P., et al. 1998, ApJ, 509, 74

Giostri, R., Vargas dos Santos, M., Waga, I., et al. 2012, JCAP, 03, 027

Gondolo, P., \& Freese, K. 2003, Phys. Rev. D, 68, 063509

Gradshteyn I. S., \& Ryzhik I. M. 2007, Tables of Integrals, Series and Products, 7th edn. (Amsterdam: Elsevier - Academic Press)
Guidry, M. 1998, Neutrino transport and large-scale convection in core-collapse processes, in Nuclear and Particle Astrophysics, eds. J. G. Hirsch, \& D. Page (Cambridge: Cambridge University Press)

Guy, J., Astier, P., Baumont, S., et al. 2007, A\&A, 466, 11

Hamuy, M., Phillips, M. M., Suntzeff, N. B., et al. 1996, AJ, 112, 2408

Hicken, M., Challis, P., Jha, S., et al. 2009a, ApJ, 700, 331

Hicken, M., Wood-Vasey, M., Blondin, S., et al. 2009b, ApJ, 700, 1097

Hinshaw G., Larson, D., Komatsu, E., et al. 2013, ApJS, 208, 19

Holtzman, J. A., Marriner, J., Kessler, R., et al. 2008, AJ, 136, 2306

Horedt, G. P. 2004, Polytropes: Aplications in Astrophysics and Related Fields (Dordrecht: Kluwer Academic Publishers)

Hooper, D. 2009, TASI 2008 Lectures on Dark Matter [arXiv : 0901.4090]

Hooper, D., Finkbeiner, D. P., \& Dobler, G. 2007, Phys. Rev. D, 76, 083012

Huterer, D. 2002, Phys. Rev. D, 65, 063001

Jaffe, A. H., Ade, P. A., Balbi, A., et al. 2001, Phys. Rev. Lett., 86, 3475

Jha, S., Kirshner, R. P., Challis, P., et al. 2006, AJ, 131, 527

Kamenshchik, A., Moschella, U., \& Pasquier, V. 2001, Phys. Lett. B, 487, 7

Kamiuto, K. 2008, Polytropic change of a closed system with a negative exponent (in Japanese), Oita University, Graduate School of Engineering, Research Report No 55, 1 http://hdl .handle.net/10559/13257

Kane, G., Lu, R., \& Watson, S. 2009, Phys. Lett. B, 681, 151

Karami, K., \& Abdolmaleki, A. 2010a, Ap\&SS, 330, 139

Karami, K., \& Abdolmaleki, A. 2010b, Phys. Scr., 81, 055901

Karami, K., \& Abdolmaleki, A. 2012, J. Phys. Conf. Ser., 375, 032009

Karami, K., \& Khaledian, M. S. 2012, Int. J. Mod. Phys. D, 21, 1250083

Karami, K., Ghaffari, S., \& Fehri, J. 2009, EPJ C, 64, 85

Kessler, R., Becker, A. C., Cinabro, D., et al. 2009, ApJS, 185, 32

Kleidis, K., \& Spyrou, N. K. 2011, A\&A, 529, A26

Klypin, A., Holtzman, J., Primack, J. R. \& Regos, E. 1993, ApJ, 416, 1

Knop, R. A., Aldering, G., Amanullah, R., et al. (Supernova Cosmology Project Group) 2003, ApJ, 598, 102

Kolb, E. W., \& Turner, M. S. 1990, The Early Universe (Menlo Park: AddisonWesley)

Komatsu, E., Dunkley, J., Nolta, M. R., et al. 2009, ApJS, 180, 330

Komatsu, E., Smith, K. M., Dunkley, J., et al. 2011, ApJS, 192, 18

Kowalski, M., Rubin, D., Aldering, G., et al. (Supernova Cosmology Project Group) 2008, ApJ, 686, 749

Krisciunas, K., Garnavich, P. M.. Challis, P., et al. 2005, AJ, 130, 2453

Landau, L. D., \& Lifshitz, E. M. 1987, Fluid Mechanics, 2nd edn. (Oxford: Pergamon Press)

Li, M. 2004, Phys. Lett. B, 603, 1

Lima, J. A. S., Silva, F. E., \& Santos, R. C. 2008, Class. Quant. Grav., 25, 205006

Lima, J. A. S., Jesus, J. F., \& Oliveira, F. A. 2010, JCAP, 11, 027

Lima, J. A. S., Basilakos, S., \& Costa, F. E. M. 2012, Phys. Rev. D, 86, 103534

Linder, E. V. 2008, Rep. Prog. Phys., 71, 056901

Lundgren A. P., Bondarescu, M., Bondarescu, R., \& Balakrishna, J. 2010, ApJ, 715, L35

Makler, M., de Oliveira, S. Q., \& Waga, I. 2003, Phys. Lett. B, 555, 1

Malekjani, M., Khodam-Mohamadi, A., \& Taji, M. 2011, Int. J. Theor. Phys., 50,3112

Masaki, S., Fukugita, M., \& Yoshida, N. 2012, ApJ, 746, 38

Meng, X. H., Ren, J., \& Hu, M. G. 2007, Commun. Theor. Phys., 47, 379

Miao L. I., Xiao-Dong, L. I., Wang, S., \& Wang Y. 2011, Commun. Theor. Phys., 56,525

Miknaitis, G., Pignata, G., Rest, A., et al. 2007, ApJ, 666, 674

Mongan, T. R. 2001, Gen. Relativ. Grav., 33, 1415

Mukhopadhyay, V., Ray, S., \& Dutta, C. S. 2008, Mod. Phys. Lett. A, 23, 3187

Narlikar, J. V. 1983, Introduction to Cosmology (Boston: Jones and Bartlett Publishers Inc.)

Netterfield, C. B., Ade, P. A. R., Bock, J. J., et al. 2002, ApJ, 571, 604

Nojiri, S., Odintsov, S. D., \& Tsujikawa, S. 2005, Phys. Rev. D, 71, 063004

Nunez, D., Sussman, R. A., Zavala, J., Cabral-Rosetti, L. G., \& Matos, T. 2006, eds. A. Bashir, V. Villanueva, L. Villasenor, M. A. Perez \& L. Urrutia, AIP Conf. Proc., 857, 316

Olive, K. A. 2003, TASI Lectures on Dark Matter [arXiv : astro-ph/0301505]

Padin, S., Cartwright, J. K., Mason, B. S., et al. 2001, ApJ, 549, L1

Padmanabhan, T. 2002, Phys. Rev. D, 66, 021301

Padmanabhan, T. 2003, Phys. Rep., 380, 235

Pavón, D., \& Zimdahl, W. 2005, Phys. Lett. B, 628, 206

Peacock, J. A. 1999, Cosmological Physics (Cambridge: Cambridge University Press)

Peccei, R. D. 2005, Phys. Rev. D, 71, 023527

Percival, W. J., Reid, B. A., Eisenstein, D. J., et al. 2010, MNRAS, 401, 2148

Perivolaropoulos, L. 2003, Phys. Rev. D, 67, 123516

Perlmutter, S., Filippenko, A. V., Challis, P., et al. (Supernova Cosmology Project Group) 1998, Nature, 391, 51 
Perlmutter, S., Aldering, G., Goldhaber, G., et al. (Supernova Cosmology Project Group) 1999a, ApJ, 517, 565

Perlmutter, S., Turner, M. S., \& White, M. 1999b, Phys. Rev. Lett., 83, 670

Ren, J., \& Meng, X. H. 2006, Phys. Lett. B, 633, 1

Regis, M., \& Ullio, P. 2008, Phys. Rev. D, 78, 3505

Riess, A. G. 2012, Rev. Mod. Phys., 84, 1965

Riess, A. G., Filippenko, A. V., Challis, P., et al. 1998, AJ, 116, 1009

Riess, A. G., Kirshner, R. P., Schmidt, B. P., et al. 1999, AJ, 117, 707

Riess, A. G., Nugent, P. E., Gilliland, R. L., et al. 2001, ApJ, 560, 49

Riess, A. G., Strolger, L.-G., Tonry, J., et al. 2004, ApJ, 607, 665

Riess, A. G., Strolger, L.-G., Casertano, S., et al. 2007, ApJ, 659, 98

Sahni, V. 2004, in The Physics of the Early Universe, ed. L. Papantonopoulos (Berlin: Springer Verlag), Lect. Notes Phys., 653, 141

Sahni, V., \& Starobinsky, A. 2000, IJMP D, 9, 373

Sami, M., Savchenko, N., \& Toporensky, A. 2004, Phys. Rev. D, 70, 123528

Sandvik, H. B., Tegmark, M., Zaldarriaga, M., \& Waga, I. 2004, Phys. Rev. D, 69,123524

Saxton C. J., \& Ferreras, I. 2010, MNRAS, 405, 77

Saxton, C. J., \& Wu, K. 2008, MNRAS, 391, 1403

Scherrer, R. J. 2004, Phys. Rev. Lett., 93, 011301

Seljak, U., Slosar, A., \& McDonald, P. 2006, J. Cosmol. Astropart. Phys., 0610, 014

Sen, A. A., \& Scherrer, R. J. 2005, Phys. Rev. D, 72, 063511

Schmidt, B. P., Suntzeff, N. B., Phillips, M. M., et al. 1998, ApJ, 507, 46

Spergel, D. N., \& Steinhardt, P. J. 2000, Phys. Rev. Lett., 84, 3760

Spergel, D. N., Verde, L., Peiris, H. V., et al. 2003, ApJS, 148, 175
Spergel, D. N., Bean, R., Doré, O., et al. 2007, ApJS, 170, 377

Spyrou, N. K. 2011, J. Phys. Conf. Ser., 283, 012035

Stefancić, H. 2005, Phys. Rev. D, 71, 084024

Stompor, R., Abroe, M., Ade, P., et al. 2001, ApJ, 561, L7

Su, K.-Y., \& Chen, P. 2009, Phys. Rev. D, 79, 128301

Suzuki, N., Rubin, D., Lidman, C., et al. (Supernova Cosmology Project Group) 2012, ApJ, 746, 85

Tegmark, M., Eisenstein, D. J., Strauss, M. A., et al. 2006, Phys. Rev. D, 74, 3507

Turner, M. S. \& White, M. 1997, Phys. Rev. D, 56, R4439

Tonry, J. L., Schmidt, B. P., Barris, B., et al. 2003, ApJ, 594, 1

Van den Aarssen, L., Bringmann, T., \& Pfommer, C. 2012, Phys. Rev. Lett., 109, 231301

Wang, Y.. \& Wang, S. 2013, Phys. Rev. D, 88, 043522

Wang, Y., Freese, K., Gondolo, P., \& Lewis, M. 2003, ApJ, 594, 25

Weinberg, S. 1972, Gravitation and Cosmology (New York: John Wiley \& Sons Inc.)

Wood-Vasey, W. M., Miknaitis, G., Stubbs, C. W., et al. 2007, ApJ, 666, 694

Xu, L., Wang, Y., \& Noh, H. 2012, Phys. Rev. D, 85, 043003

Zavala, J., Nunez, D., Sussman, R. A., Cabral-Rosetti, L. G., \& Matos, T. 2006, JCAP, 6,8

Zhu, Z. H. 2004, A\&A, 423, 421

Zimdahl, W., Schwarz, D. J., Balakin, A. B., \& Pavón, D. 2001, Phys. Rev. D, 64, 3501

Zurek, K. M. 2009, Phys. Rev. D, 79, 115002 